\title{
CR8, a Selective and Potent CDK Inhibitor, Provides Neuroprotection in Experimental Traumatic Brain Injury
}

\author{
Shruti V. Kabadi • Bogdan A. Stoica • Marie Hanscom • David J. Loane • \\ Giorgi Kharebava • Michael G. Murray II • Rainier M. Cabatbat • Alan I. Faden
}

Published online: 14 December 2011

(C) The American Society for Experimental NeuroTherapeutics, Inc. 2011

\begin{abstract}
Traumatic brain injury (TBI) induces secondary injury mechanisms, including cell cycle activation (CCA), that leads to neuronal death and neurological dysfunction. We recently reported that delayed administration of roscovitine, a relatively selective cyclin-dependent kinase (CDK) inhibitor, inhibits CCA and attenuates neurodegeneration and functional deficits following controlled cortical impact $(\mathrm{CCI})$ injury in mice. Here we evaluated the neuroprotective potential of $\mathrm{CR} 8$, a more potent second-generation roscovitine analog, using the mouse CCI model. Key CCA markers (cyclin A and B1) were significantly upregulated in the injured cortex following TBI, and phosphorylation of CDK substrates was increased. Central administration of CR8 after TBI, at a dose 20 times less than previously required for roscovitine, attenuated CCA pathways and reduced post-traumatic apoptotic cell death at $24 \mathrm{~h}$ post-TBI. Central administration of CR8, at $3 \mathrm{~h}$ after TBI, significantly attenuated sensorimotor and cognitive deficits, decreased lesion volume, and improved neuronal survival in the cortex and dentate gyrus. Moreover, unlike roscovitine treatment in the same model, CR8 also attenuated posttraumatic neurodegeneration in the CA3 region of the hippocampus and thalamus at 21 days. Furthermore, delayed systemic administration of CR8, at a dose 10 times less than
\end{abstract}

Shruti V. Kabadi and Bogdan A. Stoica contributed equally to the manuscript.

S. V. Kabadi • B. A. Stoica $(\bowtie) \cdot$ M. Hanscom $\cdot$ D. J. Loane $\cdot$

G. Kharebava $\cdot$ M. G. Murray II $\cdot$ R. M. Cabatbat • A. I. Faden Department of Anesthesiology, Center for Shock, Trauma, and Anesthesiology Research (STAR), University of Maryland School of Medicine,

Baltimore, MD 21201, USA

e-mail: bstoica@anes.umm.edu previously required for roscovitine, significantly improved cognitive performance after CCI. These findings further demonstrate the neuroprotective potential of cell cycle inhibitors following experimental TBI. Given the increased potency and efficacy of CR8 as compared to earlier purine analog types of CDK inhibitors, this drug should be considered as a candidate for future clinical trials of TBI.

Keywords Cell cycle $\cdot$ Traumatic brain injury $\cdot$ Cyclindependent kinases $\cdot$ Neurodegeneration $\cdot$ Neuroprotection . CR8

\section{Introduction}

Traumatic brain injury (TBI) is a major public health problem, with more than 1.7 million new cases reported annually in the United States [1], which accounts for half of all trauma-related deaths [2]. TBI causes cell death and neurological dysfunction through both direct physical disruption of tissue (primary injury), as well as delayed and potentially reversible molecular and cellular mechanisms that cause progressive white and grey matter damage (secondary injury) $[3,4]$. Delayed injury begins within seconds to minutes after the insult and may be responsible for a significant component of the neurodegeneration and neurological impairment that continues for days, weeks, or potentially months to years $[4,5]$.

Previous studies have linked cell cycle events and neuronal cell death. Historically, post-mitotic cells, such as neurons, were thought to have permanently entered the G0 phase and were incapable of entering the cell cycle. However, recent studies indicate that cell cycle re-entry occurs in mature differentiated post-mitotic neurons, resulting in apoptosis rather 
than neuronal proliferation [6-8]. In proliferating cells, the cell cycle is controlled by complex molecular mechanisms, and progression through distinct phases that require sequential activation of a large group of Ser/Thr kinases called the cyclindependent kinases (CDKs) and their positive regulators (cyclins) [9]. The G1 phase is initiated sequentially by increased levels of members of the cyclin D family, activation of cyclin D-dependent kinase activity, phosphorylation of the retinoblastoma $(\mathrm{Rb})$ family, and activation of the E2 promoter binding factor (E2F) family of transcription factors. Active $\mathrm{E} 2 \mathrm{~F}$ induces transcription of various genes involved in the cell cycle, such as cyclin A which associates with CDK2 [10]. In the late $\mathrm{G} 2$ phase, cyclin $\mathrm{A}$ is degraded, whereas CDK2 binds to B-type cyclins, facilitating $\mathrm{G} 2 / \mathrm{M}$ phase transition $[10,11]$. In contrast, in post-mitotic neurons the activation of E2F members may contribute to increased transcription of proapoptotic molecules, such as caspase- $3,-8$, and -9 , and Apaf-1 or anti-apoptotic Bcl-2 family members leading to cell death $[8,12,13]$. Recent evidence demonstrates neuronal cell cycle activation (CCA) following TBI, suggesting that under these conditions CCA may represent a key secondary injury mechanism that contributes to neuronal cell death [10].

We have previously investigated the neuroprotective potential of a relatively selective CDK inhibitor roscovitine, which is currently being evaluated clinically for the treatment of certain cancers [14-16]. Roscovitine attenuates CCA, progressive neurodegeneration, and neurological dysfunction in several models of TBI $[17,18]$. However, the therapeutic potential of roscovitine may be limited by several issues, including a short biological half-life, rapid metabolism to inactive derivatives, and relatively weak potency as an inhibitor of purified and cellular CDKs [16, 19, 20]. The N6-biaryl-substituted derivative of roscovitine, which is called CR8, was synthesized and optimized in an effort to generate second-generation roscovitine analogs with greater therapeutic potential compared to the parent compound [16]. In this study we evaluated the neuroprotective effects of CR8 using a well-established experimental TBI model of controlled cortical impact (CCI) in mice.

\section{Materials and Methods}

\section{Injury}

All surgical procedures and experiments were carried out in accordance with protocols approved by the Animal Care and Use Committee at the University of Maryland. Our customdesigned CCI-injury device [18, 21-23] consists of a microprocessor, controlled with a pneumatic impactor that has a 3.5-mm diameter tip. Male C57BL/6 J mice (20-25 g) were anesthetized with isoflurane (induction at $4 \%$ and maintenance at $2 \%$ ) evaporated in a gas mixture containing $70 \%$
$\mathrm{N}_{2} \mathrm{O}$ and $30 \% \mathrm{O}_{2}$, which was administered through a nose mask. Depth of anesthesia was assessed by monitoring respiration rate and pedal withdrawal reflexes. The mice were placed on a heated pad to maintain a core body temperature of $37^{\circ} \mathrm{C}$. The head was mounted in a stereotaxic frame, and the surgical site was clipped and cleaned with Nolvasan (Revival Animal Health, Orange City, IA) and ethanol scrubs. A $10-\mathrm{mm}$ midline incision was made over the skull; the skin and fascia were reflected; and a 4-mm craniotomy was made on the central aspect of the left parietal bone. The impounder tip of the injury device was then extended to its full stroke distance $(44 \mathrm{~mm})$, positioned to the surface of the exposed dura, and reset to impact the cortical surface. Moderate injury was induced using an impactor velocity of $6 \mathrm{~m} / \mathrm{s}$ and deformation depth of $2 \mathrm{~mm}$, as previously detailed [18, 22, 23]. After injury, the incision was closed with interrupted $6-0$ silk sutures, the anesthesia was terminated, and the animal was placed into a heated cage to maintain normal core temperature for 45 minutes post-injury. All animals were monitored carefully for at least 4 hours after surgery and then again daily. The systemic temperature of the mice was controlled during the injury and drug administration. Sham animals underwent the same procedure as injured mice except for the impact.

\section{Drug Administration}

\section{Study 1 (Biochemistry)}

Groups of mice ( $\operatorname{sham}[\mathrm{n}=3]$, vehicle $[\mathrm{n}=5]$, and CR8 $[\mathrm{n}=5]$ ) received an intracerebroventricular injection of (R)-CR8 (Tocris Bioscience, Ellisville, $\mathrm{MO}$ ) or an equal volume of a vehicle (physiological saline) 30 minutes after CCI injury. A 1.5- mM $(1.5 \mathrm{nmoles} / \mu \mathrm{l}) \mathrm{CR} 8$ solution in saline was injected into the left ventricle (coordinates from bregma $=$ anterior $(A):-0.5$, lateral (L): -1.0, ventral (V): -2.0) using a 30-gauge needle attached to a Hamilton syringe (Hamilton, Reno, NV) at a rate of $0.5 \mu \mathrm{l} /$ minute, with a final volume of $5 \mu \mathrm{l}$ (7.5 nmoles). This dose was based on our prior published work using the structurally similar purine analog inhibitor roscovitine in the same model [18], and pilot comparisons of the neuroprotective effects of the 2 compounds in vitro.

\section{Study 2 (Behavior/Histology)}

Groups of mice (each, $n=10$ /group) received an intracerebroventricular injection of CR8, or equal volume vehicle (physiological saline) $3 \mathrm{~h}$ after injury. A $1.5-\mathrm{mM}(1.5$ nmoles/ $\mu$ l) CR8 solution in saline was injected into the left ventricle as previously described. Sham-operated mice received an intracerebroventricular injection of a vehicle $3 \mathrm{~h}$ after surgery. 


\section{Study 3 (Behavior)}

Mice were subjected to $\mathrm{CCI}$ and randomized to receive treatment with either intraperitoneal injections of CR8 $(5 \mathrm{mg} / \mathrm{kg} ; \mathrm{n}=7)$ or an equal volume vehicle $(\mathrm{n}=8) 3 \mathrm{~h}$ after injury. Sham-operated mice received an intraperitoneal injection of a vehicle $3 \mathrm{~h}$ after surgery. The dose of CR8 administered intraperitoneal was based on our previous data comparing effective central and systemic administration doses for roscovitine in the same model [18], which was the effective intracerebroventricular dose from study 1 .

\section{Western Immunoblot}

At the appropriate time points mice (sham $[\mathrm{n}=3]$, vehicle $[\mathrm{n}=$ 5], and CR8 [ $\mathrm{n}=5]$ ) treated with CR8 or vehicle (intracerebroventricularly) were anesthetized $(100 \mathrm{mg} / \mathrm{kg}$ sodium pentobarbital intraperitoneally), transcardially perfused with ice-cold saline, and decapitated. A 5-mm area surrounding the lesion epicenter on the ipsilateral cortex was rapidly dissected and immediately frozen on dry ice. Cortical tissue was homogenized in radioimmunoprecipitation assay (RIPA) buffer and centrifuged at $15,000 \mathrm{rpm}$ for 15 minutes at $4^{\circ} \mathrm{C}$ to isolate proteins, and protein concentration was determined using the Pierce BCA Protein Assay kit (Thermo Scientific, Rockford, IL). Twenty-five $\mu \mathrm{g}$ of protein was run on sodium dodecyl sulfate (SDS) polyacrylamide gel electrophoresis and transferred onto nitrocellulose membrane. The blots were probed with antibodies against cyclin A (1:2000, Santa Cruz Biotechnology, Inc., Santa Cruz, CA), cyclin B1 (1:2000, Santa Cruz Biotechnology, Inc.), phospho-(ser) CDKs substrate (1/2000, Cell Signaling Technology, Danvers, MA), phospho-n-myc (Bethyl Laboratories, Inc, Montgomery, TX), and fodrin (1:2000, Enzo Life Sciences International, Inc., Plymouth Meeting, PA). $\beta$-actin (1:2000; Sigma-Aldrich, St. Louis, $\mathrm{MO}$ ) was used as an endogenous control. Immune complexes were detected with the appropriate horseradish peroxidase (HRP)-conjugated secondary antibodies (KPL, Inc., Gaithersburg, MD) and visualized using SuperSignal West Dura Extended Duration Substrate (Thermo Scientific, Rockford, IL). Chemiluminescence was captured on a Kodak Image Station 4000R station (Carestream Health, Rochester, NY), and protein bands were quantified by densitometric analysis using Carestream Molecular Imaging Software. The data presented reflect the intensity of the target protein band compared to the control and were normalized based on the intensity of the endogenous control for each sample (expressed in arbitrary units).

\section{Beam Walk}

Chronic motor recovery was assessed using a beam walk test as previously described $[18,21,22]$. The beam walking task discriminates fine motor coordination differences between injured and sham-operated animals. The test consists of a narrow wooden beam $(5-\mathrm{mm}$ wide and $120 \mathrm{~mm}$ in length), which is suspended $300 \mathrm{~mm}$ above a tabletop. Mice were placed on 1 end of the beam, and the number of foot faults of the right hind limb was recorded over 50 steps. Mice were trained on the beam walk for 3 days prior to TBI and tested at $1,3,7,14$, and 21 days after injury.

\section{Morris Water Maze}

Spatial learning and working memory following TBI was assessed using the acquisition paradigm of Morris water maze (MWM) test on post-injury days 14, 15, 16, and 17, as previously described [18, 21-23]. A white circular pool was divided into 4 quadrants using computer-based AnyMaze video tracking system (Stoelting Co., Wood Dale, IL) and the platform was hidden in 1 quadrant (southwest), 14 inches from the sidewall. Spatial learning and memory performance was assessed by determining the latency (seconds) to locate the submerged hidden platform with a 90second limit per trial. Reference spatial memory was assessed by a probe trial, with a 60 -second limit, on postinjury day 18 . The latency to the first entry and number of entries into the target quadrant where the platform had been hidden during the acquisition phase were recorded. A visual cue test was subsequently performed on post-injury day 18 , using a flag placed on the platform in 1 of the quadrants (with a 90-second limit per trial), and latency (seconds) to locate the flagged platform was recorded. Water maze search strategy analysis was performed, as previously described [24]. Three strategies were identified using a categorization scheme: 1) spatial strategies were defined as swimming directly to platform with no more than 1 loop, or swimming directly to the correct target quadrant and searching; 2) systematic strategies were defined as searching the interior portion or the entire tank without spatial bias, and searching incorrect target quadrant; and 3) looping strategies were defined as circular swimming around the tank, swimming in a tight circle, and swimming around the wall of the tank. The search strategies were analyzed on all acquisition trials on post-injury day 17 , and a percentage of each strategy in each group was calculated.

\section{Novel Object Recognition}

Retention or intact memory was assessed by the novel object recognition (NOR) test on post-injury day 21 . The apparatus consists of an open field $(22.5 \mathrm{~cm} \times 22.5 \mathrm{~cm})$ with 2 adjacently located imaginary circular zones, as previously designed $[23,25]$. The zones are equally spaced from the sides in the center of the square and designated as "old object" and "novel object" zones, using the AnyMaze video tracking system. The old object used was square-shaped, 
whereas the novel object was L-shaped, and assembled by building blocks from Lego toys (Lego, Enfield, CT), which were clearly distinct in shape and appearance. On postinjury day 20, all animals were placed in the open field for 5 minutes each, without any objects present for habituation. Two 5-minute trials were performed on post-injury day 21 ; the first (training) trial was performed with 2 old objects in both zones and the second (testing) phase with 1 old object and 1 novel object present in the respective zones of the open field. There was an inter-trial interval of 60 minutes, during which the animals were returned to their home cages. Retention memory was determined as novel object exploration time and the "discrimination index" (DI) for the second trial, which was calculated using the following formula:

$\%$ DI $=$ Time spent in novel object zone $\times 100$

(time spent in old object zone + time spent in novel object zone)

Immunohistochemistry

At $24 \mathrm{~h}$ (study 1) or 21 days (study 2) after injury, mice ( $\mathrm{n}=3$ / group) treated with $\mathrm{CR} 8$ or a vehicle (intracerebroventricularly) were anesthetized and transcardially perfused with saline and $10 \%$ buffered formalin phosphate solution (containing $4 \%$ paraformaldehyde) (Fisher Scientific, Pittsburg, PA). The brains were removed, post-fixed in paraformaldehyde for $24 \mathrm{~h}$ and protected in $30 \%$ sucrose. Frozen brain sections (60 $\mu \mathrm{m}$ and $20 \mu \mathrm{m}$ ) were cut on a cryostat and mounted onto glass slides. Selected slides were with stained, with Fluoro-Jade B (Chemicon, Temecula, CA) to identify degenerating neurons (study 1), or were processed for unbiased assessment of lesion volume and neuronal cell loss (study 2).

\section{Lesion Volume Assessment}

Sections were stained with cresyl violet (FD NeuroTechnologies, Baltimore, MD), dehydrated and mounted for analysis ( $n=10$ /group). Lesion volume was quantified based on the Cavalieri method of unbiased stereology using Stereologer 2000 program software (Systems Planning and Analysis, Alexandria, VA). The lesion volume was quantified by outlining the missing tissue on the injured hemisphere using the Cavalieri estimator with a grid spacing of $0.1 \mathrm{~mm}$. From 96 total $60-\mu \mathrm{m}$ sections, every eighth section was analyzed beginning from a random start point.

Assessment of Neuronal Cell Loss in Hippocampal Subregions

Stereo investigator software (MBF Biosciences, Williston, VT) was used to count the total number of surviving neurons in the Cornu Ammonis (CA)1, CA2, CA3, and dentate gyrus (DG) subregions of the hippocampus using the optical fractionator method of unbiased stereology ( $\mathrm{n}=5 /$ group). Every fourth $60-\mu \mathrm{m}$ section between $-1.22 \mathrm{~mm}$ and $-2.54 \mathrm{~mm}$ from bregma was analyzed, beginning from a random start point. The optical dissector had a size of $50 \mu \mathrm{m}$ by $50 \mu \mathrm{m}$ in the $\mathrm{x}$ - and $\mathrm{y}$-axis, respectively, with a height of $10 \mu \mathrm{m}$ and a guard-zone of $4 \mu \mathrm{m}$ from the top of the section. The sampled region for each hippocampal subfield was demarcated in the injured hemisphere and cresyl violet neuronal cell bodies were counted. For the CA1, CA2, and CA3 subregions a grid spacing of $75 \mu \mathrm{m}$ in the $\mathrm{x}$-axis and $100 \mu \mathrm{m}$ in the $\mathrm{y}$-axis was used, resulting in an area fraction of one-twelfth. For the DG subregion, a grid spacing of $175 \mu \mathrm{m}$ in the $\mathrm{x}$-axis and $100 \mu \mathrm{m}$ in the $y$-axis was used, resulting in an area fraction of one-twenty-eighth. The volume of the hippocampal subfield was measured using the Cavalieri estimator method with a grid spacing of $50 \mu \mathrm{m}$. The estimated number of surviving neurons in each field was divided by the volume of the region of interest to obtain the neuronal cellular density, expressed as counts $/ \mathrm{mm}^{3}$.

\section{Assessment of Neuronal Cell Loss in the Cortex} and Thalamus

The total number of surviving neurons was quantified in the cortex and thalamus using the optical fractionator method of unbiased stereology, as previously described ( $n=5 /$ group). The optical dissector had a size of $50 \mu \mathrm{m}$ by $50 \mu \mathrm{m}$ in the $\mathrm{x}$ and y-axis, respectively with a height of $10 \mu \mathrm{m}$ and a guardzone of $4 \mu \mathrm{m}$ from the top of the section. A grid spacing of $400 \mu \mathrm{m}$ in the $\mathrm{x}$-axis and $400 \mu \mathrm{m}$ in the $\mathrm{y}$-axis was used, resulting in an area fraction of 164th. The estimated number of surviving neurons in each field was divided by the volume of the region of interest to obtain the neuronal cellular density, expressed as counts $/ \mathrm{mm}^{3}$.

\section{Statistical Analysis}

The number of animals per group for each assessment was based on our prior studies using the CCI model, which satisfied the power requirements. The data for expression of biochemical markers using Western blotting were analyzed by one-way analysis of variance (ANOVA) followed by post-hoc adjustments using the Student-Newman-Keuls test. Lesion volume, functional data, and unbiased stereological analysis were performed by an investigator blinded to the groups. Quantitative data were expressed as mean \pm standard errors of the mean. Functional data (foot faults and latency to find the platform) for beam walk and acquisition phase of MWM, respectively, were analyzed by repeated measures (trial/time) one-way ANOVA (groups) to determine the interactions of post-injury days and groups, followed by post-hoc adjustments using the Student-NewmanKeuls test. The comparisons of search strategies used to 
locate the platform on post-injury day 17 between groups were performed by chi-square $\left(\chi^{2}\right)$ analysis. The data for the probe trial of the MWM and NOR tests and stereological assessments were analyzed by one-way ANOVA followed by post-hoc adjustments using the Student-Newman-Keuls test. The data for lesion volume quantification in treated animals were compared to the vehicle-treated control using a one-tailed paired Student's $t$ test. Regression analysis between functional improvement (motor and cognition) and stereological assessment (lesion volume and CA3/DG neuronal cell loss, respectively) was performed by linear regression modeling, followed by determination of statistical significance and a correlation coefficient $\left(\mathrm{r}^{2}\right)$ to confirm the goodness of fit. The functional data was analyzed using SigmaPlot 12 (Systat Software, San Jose, CA). All other statistical tests were performed using the GraphPad Prism Program (version 3.02 for Windows; GraphPad Software, San Diego, CA). A $p<0.05$ was considered statistically significant.

\section{Results}

Up-regulation of Cyclins A and B1, and Activation of CDK Signaling following TBI

To demonstrate CCA after TBI, we evaluated the expression of cyclins A and B1 in the injured cortex by Western blot analysis. There was significant up-regulation of cyclin A (Fig. 1a, b; $p<0.01$ vs sham) and cyclin B1 (Fig. 1a, c; $p<$ $0.05 v s$ sham) expression levels at $6 \mathrm{~h}$ after TBI. The expression of cyclins A and B1 returned to control levels by $24 \mathrm{~h}$. To determine the nature of TBI-induced changes in CDK activity, we used an antibody that recognizes the phosphorylated CDKs motif, phospho-(Ser) CDKs substrate, in Western blot analysis of injured cortical tissue. We observed a significant increase in phospho-(Ser) CDKs substrate levels at $24 \mathrm{~h}$ after TBI (Fig. 1d, e; $p<0.05$ vs sham).

\section{Central Administration of CR8 Inhibits Cell Cycle Activation and Apoptosis after TBI}

To evaluate the effect of CR8 treatment on post-traumatic CCA, TBI-injured mice were administered CR8 or a vehicle by intracerebroventricular injection at 30 minutes post-TBI, and cortical tissue was collected at $6 \mathrm{~h}$ post-TBI for Western blot analysis. Cyclin A and cyclin B1 expression was significantly increased at $6 \mathrm{~h}$ after TBI (Fig. 2a, b [cyclin A]; 2a, 2c [cyclin B1]; $p<0.001$ vs sham). Notably, CR8 treatment significantly attenuated the expression of both cyclins A and B1 ( $p<0.01$ and $p<0.05$, respectively, vs vehicle). Cyclins $\mathrm{A}$ and B1 activate CDK1; therefore, to examine its activity, we measured the levels of phospho-n-myc, a CDK1 substrate. Our data demonstrated increased levels of phospho-n-myc following TBI (Fig. 2a, d; $p<0.001 v s$ sham); CR8 treatment significantly attenuated these changes ( $p<0.01 v s$ vehicle). To assess the effect of CR8 on markers of apoptosis, the presence of cleaved fragments of fodrin [26, 27] was assessed in these samples. TBI significantly increased fodrin cleavage (Fig. 2a, e; $p<0.001 v s$ sham), as demonstrated by increased levels of the $145 / 150 \mathrm{kDa}$ cleavage product. Notably, CR8 treatment significantly decreased the level of the $145 / 150 \mathrm{kDa}$ product when compared with vehicle-treated samples $(p<0.01$ vs vehicle).

Central Administration of CR8 Significantly Improves Functional Recovery following TBI

To evaluate the neuroprotective potential of CR8 against TBI-induced functional impairments, we used a delayed and more clinically relevant treatment paradigm in which CR8 (1.5 mM) was administered by intracerebroventricular injection at $3 \mathrm{~h}$ post-injury to CCI-injured mice and was compared with vehicle (saline)-treated mice. The TBI mice were followed for 21 days in functional testing after which brain tissue was collected for histological assessment.

Functional assessment of fine motor coordination was performed at various time points after injury using a beam walk test. The interaction of "post-injury days $\mathrm{X}$ groups" $(\mathrm{F}(8,110)=2.833 ; p=0.007)$ was statistically significant, and TBI induced significant sensorimotor impairments at all time points when compared with sham-injured mice (Fig. $3 ; p<0.001$ vs sham). CR8-treated mice exhibited significant improvements in sensorimotor performance at $7(p=$ $0.002 v s$ vehicle), 14 ( $p=0.014 v s$ vehicle), and 21 ( $p=$ $0.002 v s$ vehicle) days after injury when compared with vehicle-treated mice.

Spatial learning was tested using the acquisition phase of the MWM test. The interaction of "post-injury days $\mathrm{X}$ groups" $(\mathrm{F}(6,100)=0.883 ; p=0.510)$ was not statistically significant. The factors of "post-injury days" $(\mathrm{F}(3,100)=$ $3.6 ; p=0.016)$ and "groups" $(\mathrm{F}(2,100)=21.75 ; p<0.001)$ were found to be significant. TBI resulted in learning impairments on 15, 16, and 17 days after injury (Fig. 4a) $(p<0.01$ vs sham). CR8-treated mice showed improvements in cognitive performance with significantly reduced latency to find the submerged platform on post-injury day 15 ( $p=0.017 v s$ vehicle) and post-injury day $17(p=0.0015$ $v s$ vehicle) when compared with vehicle-treated mice.

Reference memory was assessed using the MWM probe trial on post-injury day 18 . TBI caused significant cognitive impairments in this test when compared with sham-injured mice (Fig. 4b, c; $p<0.05$ vs sham). CR8-treated mice exhibited significant improvements in cognitive performance in terms of number of entries (Fig. 4b; $p<0.05 v s$ vehicle) and latency to the first entry into the target quadrant (Fig. 4c; $p<$ $0.001 v s$ vehicle) when compared with vehicle-treated mice. 
Fig.1 Traumatic brain injury (TBI) induces up-regulation of cyclins A and B1 and cyclinactivation. (a-c) The expression of the 2 key cyclins (A and B1) was evaluated in cortical tissue following controlled cortical impact (CCI) by Western blot analysis. There was a significant up-regulation of cyclin $\mathrm{A}(\mathbf{a}, \mathbf{b})$ $\left({ }^{* *} p<0.01 v s\right.$ sham) at $6 \mathrm{~h}$, followed by a reduction at $24 \mathrm{~h}$ post-injury $(\hat{p}<0.05 v s 6$ $\mathrm{h}$ injured samples). The expression of cyclin B1 $(\mathbf{a}, \mathbf{c})\left({ }^{*} p<0.05\right.$ $v s$ sham) was significantly increased at $6 \mathrm{~h}$ after TBI. (d, e) To determine TBI-induced changes in CDK activity, levels of phospho-(Ser)-CDK substrates were evaluated. We observed a significant increase in phospho(Ser)-CDK substrate levels ( ${ }^{*} p<0.05$ vs sham; $\hat{p}<0.05$ vs 6-h injured samples) at $24 \mathrm{~h}$ after TBI. Representative Western blots are shown. Analysis by one-way analysis of variance, followed by post-hoc adjustments using the StudentNewman-Keuls test. Mean \pm standard error of the mean ( $n=3-5 /$ group) dependent kinase (CDK)

a

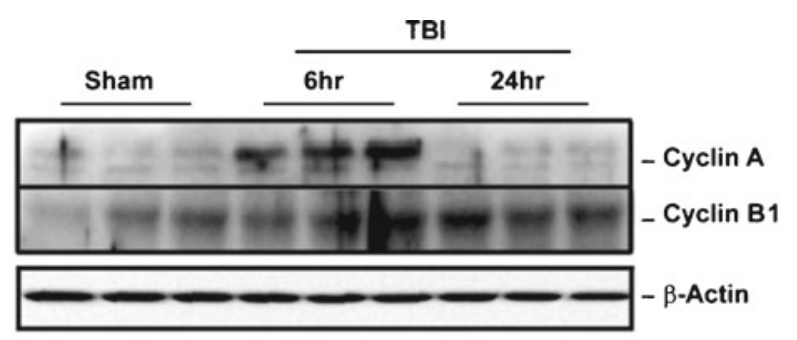

b

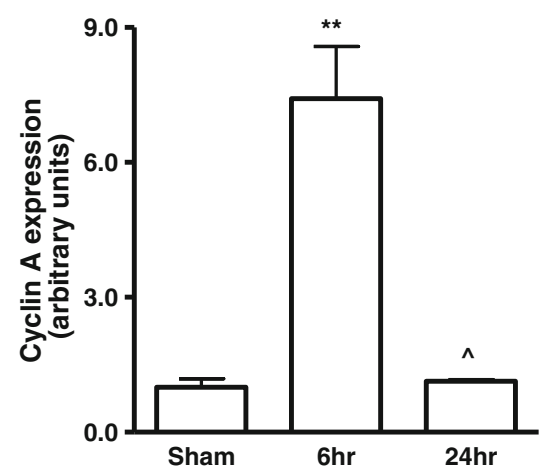

C

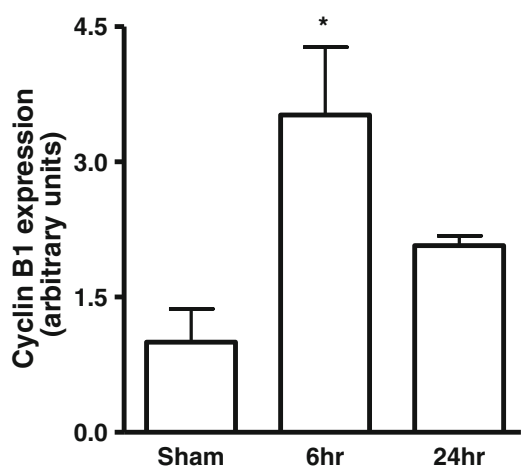

d TBI

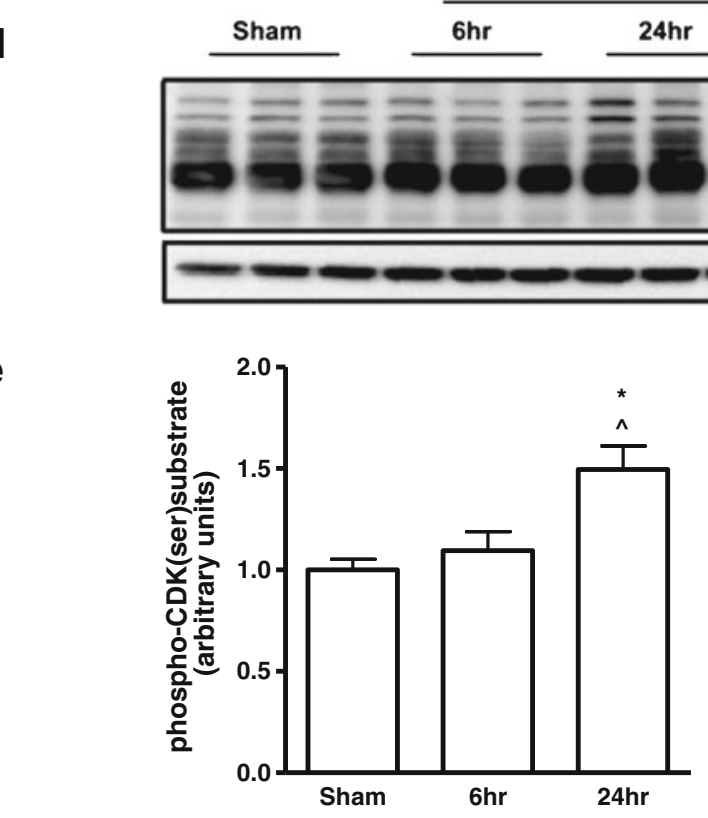

To assess the strategies used by the mice to find the hidden platform on post-injury day 17 , the swimming pattern for each mouse was analyzed and assigned a search strategy (spatial, systematic, or looping). Vehicle-treated TBI mice exhibited significantly higher reliance on looping strategies than spatial and systematic behavior (Fig. 4d; $p<0.001$ vs sham; $\chi^{2}=$ 37.64). CR8-treated TBI mice showed significantly reduced usage of looping strategy and increased reliance on spatial and

systematic search strategies $\left(p<0.001 v s\right.$ vehicle; $\left.\chi^{2}=37.64\right)$. All mice performed well in the visual cue test (Fig. 4e, d), and swim speeds did not differ across groups (Fig. 4f).

Retention or intact memory was evaluated using the NOR test. TBI caused significant cognitive impairments in this test when compared with sham-injured mice (Fig. 5a, b; $p<0.01$ vs sham). CR8 caused significant improvements in memory performance in terms of novel object exploration time 
Fig. 2 Central administration of CR8 inhibits cell cycle activation and apoptosis in cortical tissue after traumatic brain injury (TBI). (a-g) The effect of CR8 treatment on post-traumatic cell cycle activation (CCA) was evaluated by Western blot analysis of injured cortical tissue at $6 \mathrm{~h}$ after TBI. A significant increase resulted in cyclin A expression from TBI $(\mathbf{a}, \mathbf{b})$ ${ }^{* * *} p<0.001$ vs sham), whereas CR8 treatment significantly attenuated the TBI-induced increase $\left({ }^{++} p<0.01 v s\right.$ vehicle). TBI significantly increased $\left({ }^{* * *} p<0.001 v s\right.$ sham) cyclin B1 expression (a, c), and the levels of the CDK1 substrate phosphon-myc (a, d); CR8 treatment significantly attenuated levels of both CCA markers (cyclin B1: ${ }^{+} p<0.05$ vs vehicle; phospho-n-myc: ${ }^{++} p<0.01 v s$ vehicle). To be able to assess the effect of CR8 on apoptosis, the presence of $145 / 150 \mathrm{KDa}$ cleaved fragment of fodrin was determined. TBI increased levels of the $145 / 150 \mathrm{kDa}$ fodrin fragment (a, e) $\left(^{* * *} p<0.001 v s\right.$ sham); CR8 treatment significantly the TBI-induced increase $(p<0.01$ vs vehicle). Representative Western blots are shown. Analysis by one-way analysis of variance, followed by post-hoc adjustments using the StudentNewman-Keuls test. Mean \pm standard error of the mean ( $n=3-5$ /group) a TBI
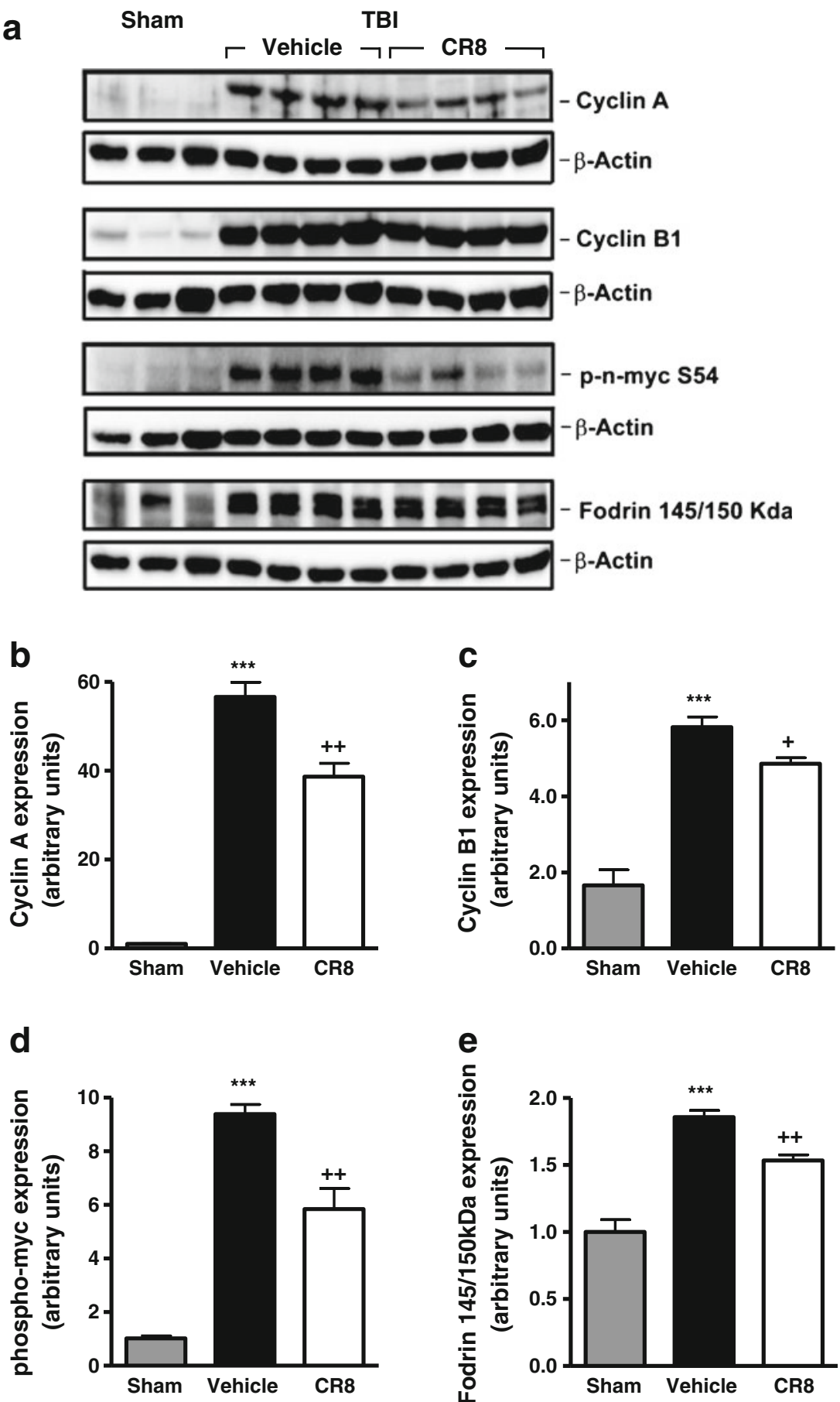

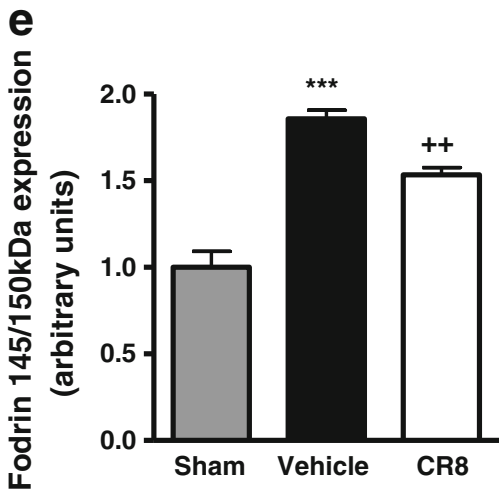

(Fig. 5a; $p<0.05 v s$ vehicle) and DI (Fig. 5b; $p<0.05 v s$ vehicle).

Central Administration of CR8 Reduces Lesion Volume following TBI

TBI-induced lesion volume was measured by unbiased stereological techniques. Histological assessment showed that vehicle-treated mice developed a large lesion following TBI (Fig. 6a, b; $14.73 \pm 0.53 \mathrm{~mm}^{3}$ ), whereas CR8 treatment resulted in a significant reduction in lesion size $\left(p<0.001\right.$ vs vehicle; $\left.8.32 \pm 0.64 \mathrm{~mm}^{3}\right)$. There was a significant positive correlation between improved sensorimotor performance in the beam walk task and reduced lesion size due to CR8 treatment following TBI (Fig. 6c; $p<0.0001 ; \mathrm{r}^{2}=0.96$ ). 


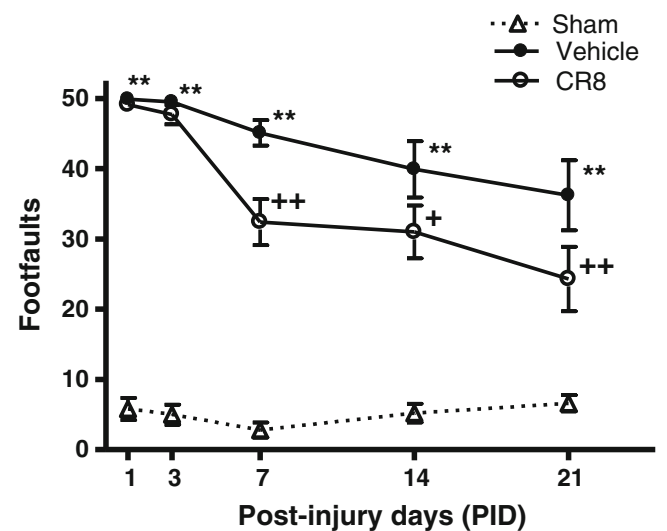

Fig. 3 Central administration of CR8 improves sensorimotor function following traumatic brain injury (TBI). Fine motor coordination deficits were quantified using the beam walk test. Hind-limb foot placement was recorded and the number of mistakes (foot faults) was recorded from 50 steps. TBI induced significant impairment in motor outcomes at all time points $\left({ }^{* *} p<0.01 v s\right.$ sham). There was a statistically significant "post-injury day $\mathrm{X}$ groups" interaction $(\mathrm{F}(8,110)=$ 2.833) $(p=0.007)$. Central administration of CR 8 at $3 \mathrm{~h}$ post-injury significantly improved fine motor coordination at 7 days $\left(^{++} p=0.002\right.$ $v s$ vehicle), 14 days $\left({ }^{+} p=0.014 v s\right.$ vehicle), and 21 days $\left({ }^{++} p=0.002 v s\right.$ vehicle) post-TBI. Analysis by repeated measures one-way analysis of variance, followed by post-hoc adjustments using the StudentNewman-Keuls test. Mean \pm standard error of the mean ( $\mathrm{n}=10$ /group)

Central Administration of CR8 Reduces Neuronal Cell Loss in the CA3 and Dentate Gyrus Subregions of the Hippocampus after TBI

Stereological assessment of surviving neurons was performed in CA1, CA2, CA3, and DG subregions of the hippocampus at 21 days after TBI. Cresyl violet staining enables discrimination of morphological features of neurons and glial cells [28,29]. Cresyl violet staining of neuroglial cells is confined to their nuclei, whereas neurons stain within the cell body, thereby enabling easy identification of neurons (Fig. 7c). TBI resulted in significant neuronal cell loss in all the hippocampal subregions $(p<0.01 v s$ sham). Central administration of CR8 after TBI failed to protect CA1 and CA2 neurons (data not shown), but resulted in significantly improved neuronal survival in the CA3 $\left(596.656 .86 \pm 50,379.58\right.$ counts $/ \mathrm{mm}^{3} ; p<$ $0.001 v s$ vehicle) (Fig. 7a, c) and DG $(649,323.14 \pm 57,443.53$ counts $/ \mathrm{mm}^{3} ; p<0.01 v s$ vehicle) (Fig. $7 \mathrm{~b}$ ) at 21 days after injury when compared with vehicle-treated samples (CA3: $229,560.90 \pm 32,067.87$ counts $/ \mathrm{mm}^{3}$, and DG: $365,908.04 \pm$ $45,142.10$ counts $/ \mathrm{mm}^{3}$ ). There was a strong, positive correlation between improved cognitive performance in the MWM test and the NOR test, and reduced neuronal cell loss in the CA3 subregion of the hippocampus due to CR8 treatment following TBI $\left(\mathrm{r}^{2}=0.94\right.$ and $\mathrm{r}^{2}=0.96$, respectively; $p<$ 0.0001) (Fig. 7d, e). Similarly, there was a positive correlation between improved cognitive performance in the MWM test and the NOR test, and reduced neuronal cell loss in the DG due to CR8 treatment following TBI $\left(\mathrm{r}^{2}=0.95\right.$ and $\mathrm{r}^{2}=0.96$, respectively; $p<0.0001$ ) (Fig. 7f, g).

\section{Central Administration of CR8 Reduces Neuronal Degeneration and Neuronal Cell Loss in the Cortex and Thalamus after TBI}

To determine the effects of CR8 treatment on TBI-induced neurodegeneration, we performed Fluoro-Jade B staining at $24 \mathrm{~h}$ after TBI (Fig. 8a, b). Representative confocal (a) and higher magnification images (Figs. 8a, 1 and 2) from the injured hemisphere in vehicle-treated mice indicate a large number of degenerating (Fluoro-Jade B positive) neurons located around the lesion site (Figs. 8a, 2) and in subcortical regions (Figs. 8a, 1). In contrast, CR8-treated mice (Fig. 8b) had less intense Fluoro-Jade B staining around the lesion (Figs. 8b, 4) and subcortical regions (Figs. 8b, 3), suggesting fewer degenerating neurons.

Stereological assessment of surviving neurons was performed in the cortex and thalamus at 21 days after TBI (Fig. 8c, d). TBI resulted in significant neuronal cell loss in the cortex and thalamus (Fig. $8 \mathrm{c}, \mathrm{d} ; p<0.01 v s$ sham). Central administration of CR8 significantly improved neuronal survival in the cortex $(146,883.0 \pm 20,531.15 \mathrm{vs}$ $87,951.28 \pm 11,684.33$ counts $/ \mathrm{mm}^{3}$ for CR8-treated and vehicle-treated samples, respectively; $p<0.05$ vs vehicle) (Fig. 8e) and thalamus $(107,031.20 \pm 6,134.16$ vs $49,861.67 \pm 6,098.05$ counts $/ \mathrm{mm}^{3}$ for CR8- and vehicletreated samples, respectively; $p<0.001 v s$ vehicle) (Fig. $8 \mathrm{f}$ ) when compared with vehicle-treated samples.

Systemic Administration of CR8 Significantly Improves Cognitive Recovery after TBI

A separate group of animals was used to investigate the therapeutic potential of delayed systemic administration of CR8 following TBI. There was significant sensorimotor impairment in the beam walk test after TBI injury (Fig. 9a) $(p<0.001$ vs sham). Although fewer foot faults were observed after CR8 treatment at all post-injury time points compared to vehicle-treated controls, these differences did not reach significance. TBI resulted in learning impairments in acquisition trials of MWM test on day 17 after injury (Fig. 9b) $(p<0.01 v s$ sham $)$. The factors of "post-injury days" $(\mathrm{F}(3,84)=$ $10.125 ; p<0.001)$ and "groups" $(\mathrm{F}(2,84)=7.931$; $p<0.001)$ were highly significant. Systemic administration of CR8 improved cognitive performance compared to controls, with significantly reduced latency to find the submerged platform on post-injury day 17 ( $p=0.022$ vs vehicle). On the probe trial of the MWM test on post-injury day 18, CR8-treated mice exhibited 
a

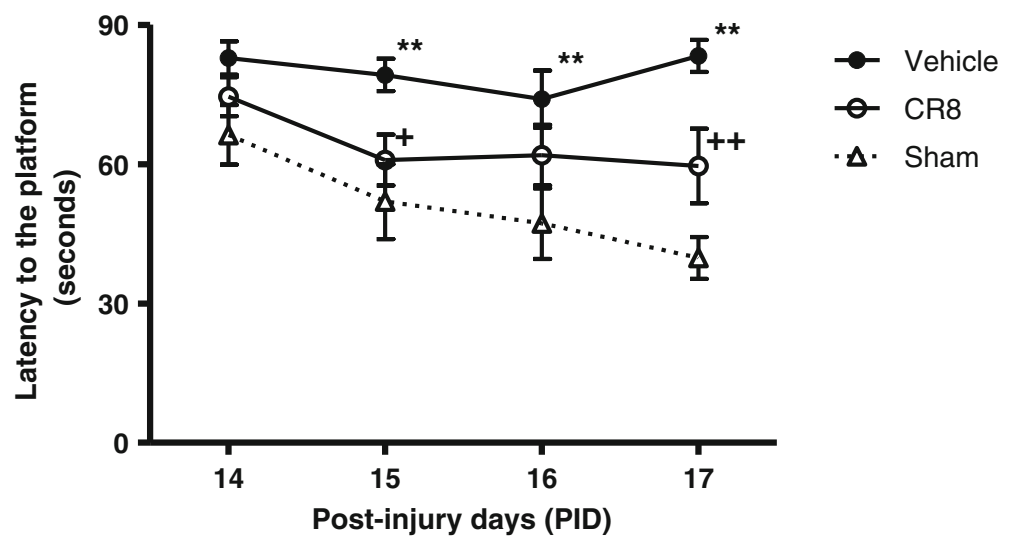

$\square$ Spatial $\boldsymbol{Z}$ Systematic

b

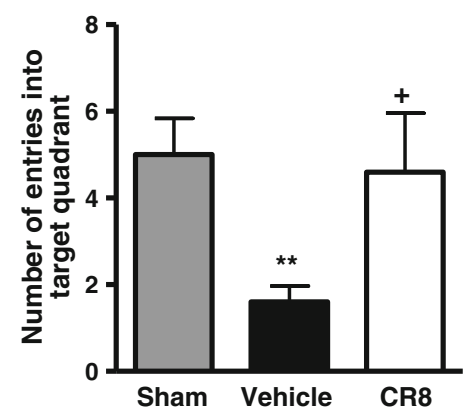

C

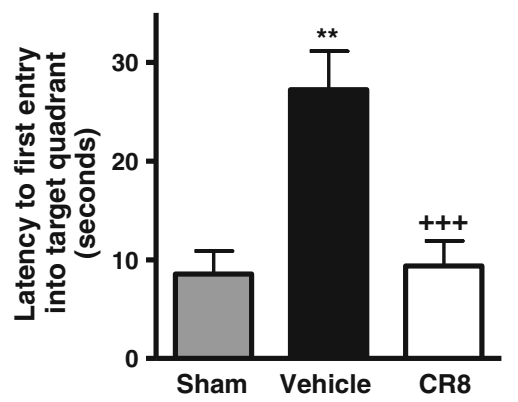

d

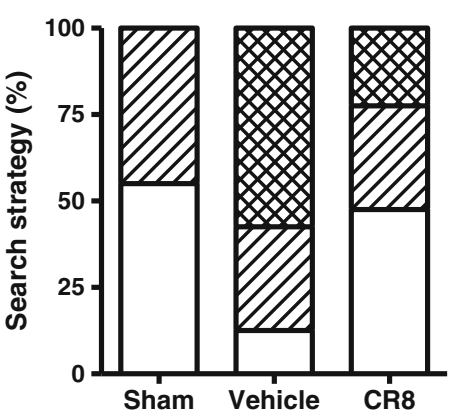

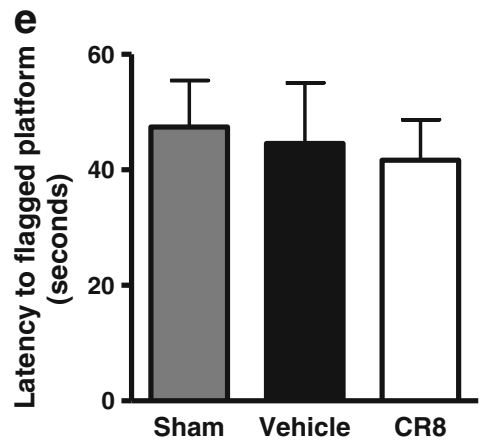

Fig. 4 Central administration of CR8 improves cognitive performance in the Morris water maze test following traumatic brain injury (TBI). (a) Spatial learning and memory was assessed using the Morris water maze (MWM) test. The factors of "post-injury days" $(\mathrm{F}(3,100)=3.6)$ $(p=0.016)$ and "groups" $(\mathrm{F}(2,100)=21.75)(p<0.001)$ were statistically significant. TBI induced significant cognitive impairments at postinjury days 15,16 , and $17\left({ }^{* *} p<0.01\right.$ vs sham). CR8-treated TBI mice had reduced latency to locate the submerged platform at day $15\left({ }^{+} p=\right.$ $0.017 v s$ vehicle) and day 17 post-TBI $\left({ }^{++} p=0.0015 v s\right.$ vehicle $)$ when compared to vehicle-treated TBI mice. Analysis by repeated measures one-way analysis of variance, followed by post-hoc adjustments using the Student-Newman-Keuls test. Mean \pm standard error of the mean $(n=10$ /group. (b, c) Reference memory was assessed using the probe trial of MWM test. TBI caused significant cognitive impairments in this test $\left({ }^{* *} p<0.05 v s\right.$ sham). CR8-treated TBI mice exhibited significant cognitive improvements in terms of number of entries (b) $\left(^{+} p<\right.$

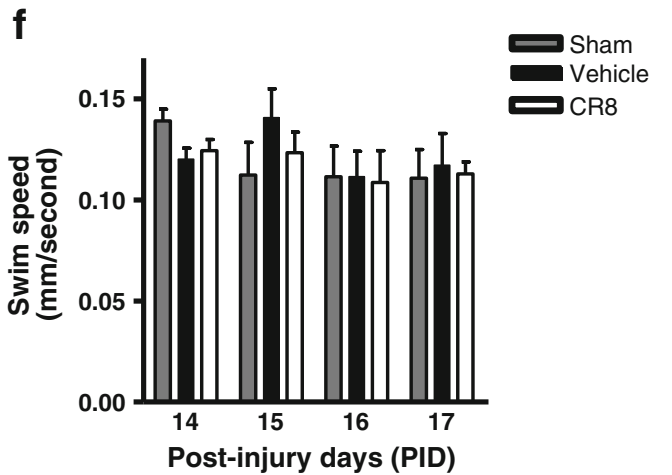

$0.05 v s$ vehicle) and latency to the first entry into the target quadrant (c) $\left({ }^{++} p<0.001 v s\right.$ vehicle) when compared to vehicle-treated TBI mice. Analysis by one-way analysis of variance, followed by post-hoc adjustments using the Student-Newman-Keuls test. (d) Cognitive performance was further evaluated by swim strategy analysis. Vehicletreated TBI mice exhibited higher reliance on looping strategies rather than spatial and systematic patterns $\left(p<0.001\right.$ vs sham; $\left.\chi^{2}=37.64\right)$. CR8-treated TBI mice showed significantly reduced usage of looping strategy and increased reliance on spatial and systematic swim strategies $\left(p<0.001 v s\right.$ vehicle; $\left.\chi^{2}=37.64\right)$. (e) The visual acuity of mice was evaluated by performing a visual cue test on post-finjury day 18 . All mice performed well and there were no statistically significant differences between the groups. (f) The swim speeds $(\mathrm{mm} / \mathrm{s})$ of all mice was recorded all throughout the test and there were no statistically significant differences between the groups on all days 

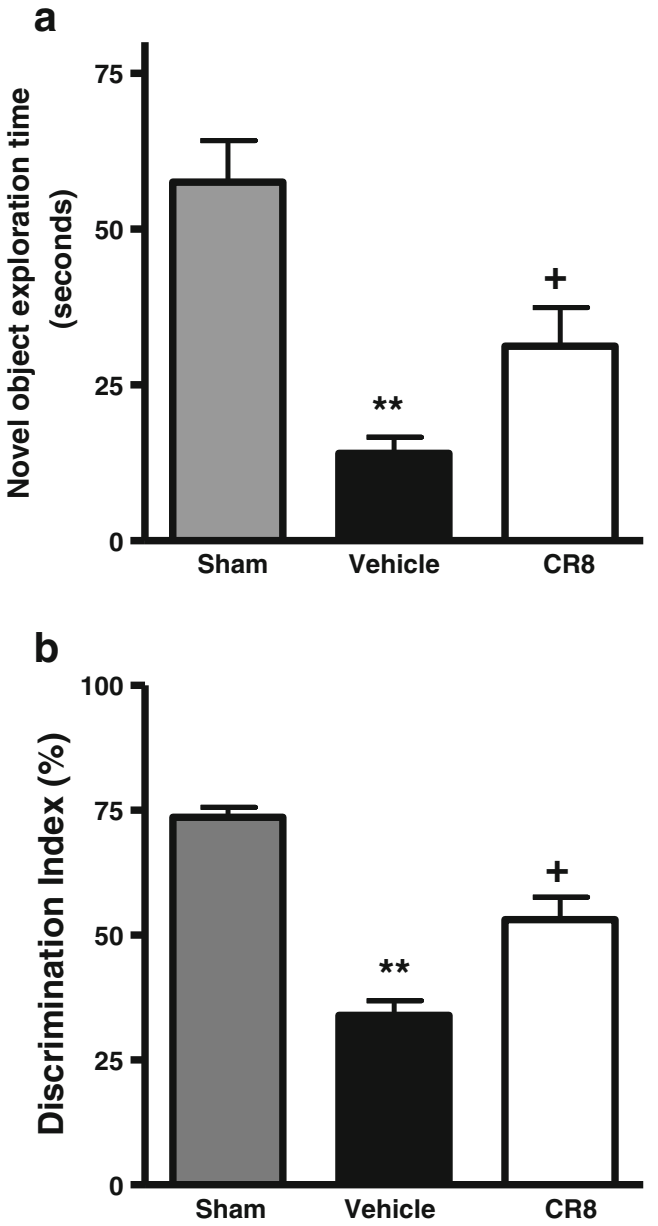

Fig. 5 Central administration of CR8 improves cognitive performance in the novel object recognition task following traumatic brain injury (TBI). (a, b) Retention or intact memory was assessed using the novel object recognition test. Vehicle-treated TBI mice showed significant cognitive impairments in this test $\left({ }^{* *} p<0.01\right.$ vs sham). CR8-treated TBI mice had significant improvements in cognitive performance in terms of novel object exploration time (f) $\left(_{p}^{+}<0.05 v s\right.$ vehicle) and discrimination index (g) $\left({ }^{+} p<0.05 v s\right.$ vehicle) when compared to vehicle-treated TBI mice. Analysis by one-way analysis of variance followed by post-hoc adjustments using the Student-Newman-Keuls test. Mean \pm standard error of the mean ( $\mathrm{n}=10$ /group)

significant improvement in cognitive performance in terms of number of entries (Fig. 9c) $(p<0.01 v s$ vehicle) when compared with vehicle-treated mice. The analysis of swimming strategies used by mice to find the hidden platform on post-injury day 17 indicated that vehicle-treated mice exhibited significantly higher reliance on looping strategies than spatial and systematic behavior (Fig. 9e) $(p<0.001 v s$ sham; $\left.\chi^{2}=23.34\right)$. CR8-treated mice showed significantly reduced usage of looping strategy and increased reliance on spatial and systematic search strategies $(p<$ 0.001 vs vehicle; $\chi^{2}=23.34$ ). TBI also caused significant cognitive impairment in the novel object recognition test on post-injury day 21 when compared with sham-injured mice
(Fig. 9f) ( $p<0.01 v s$ sham). CR8 caused significant improvement in memory performance in the NOR test $(p<0.05$ vs vehicle).

\section{Discussion}

The involvement of CCA in the pathophysiology of the central nervous system (CNS) injury is well-established [8, $11,17,18,23,30-32]$. CCA reflects a common therapeutic target for the treatment of neurodegeneration and cancer, as the cell cycle appears to be a key pathophysiological mechanism for both neuronal cell death and uncontrolled proliferation of tumor cells. Indeed, most pharmacological inhibitors for CCA that have been examined in the CNS were initially studied in oncology. More than $140 \mathrm{CDK}$ inhibitors have been described, of which 10 are currently undergoing phase 1 or phase 2 clinical trials as anti-cancer agents [19]. Roscovitine (also known as CYC202 or seliciclib) is a 2,6,9-tri-substituted purine that is in late phase 2 trials for non-small cell lung cancer and nasopharyngeal cancer $[14,15,19]$. Roscovitine has demonstrated selectivity for certain kinases, including CDKs- $1,-2,-5,-7$, and -9 ; other potentially attractive features are its oral bioavailabilty and more modest toxicity than other such inhibitors [19]. Previously, we demonstrated the neuroprotective effects of roscovitine following experimental TBI [17, 18]. Central administration of roscovitine $3 \mathrm{~h}$ post-injury decreased lesion volume and improved behavioral outcomes [18]. However, the therapeutic potential of roscovitine is limited by a relatively short elimination half-life (less than 10 minutes), and rapid metabolic inactivation [20]. In addition, its potency for inhibition of purified CDKs and CDK activity in cell lines is relatively weak [16, 33]. CR8 (an N6-biarylsubstituted derivative of roscovitine) was synthesized and optimized in an effort to generate second-generation roscovitine analogs with greater therapeutic potential compared to the parent compound [16]. CR8 exhibits a 50-fold higher potency than roscovitine in different cell lines, possibly reflecting enhanced inhibition of purified CDKs-1, -2, -5, 7 , and -9 , as well as improved cell permeability, intracellular stability, and solubility $[16,19]$.

$\mathrm{CCI}$ injury is an experimental TBI model that recapitulates certain features of clinical TBI and produces a focal injury, resulting in significant pathophysiological alterations, such as vascular disruption, cerebral edema, elevated intracerebral pressure, as well as significant long-term neurological deficits $[34,35]$. The CCI model has been extensively used to investigate the molecular and cellular events that occur during secondary injury and to evaluate novel therapeutic approaches $[22,36,37]$. Based on previous studies [16, 19] and our pilot in vitro data (not shown) indicating a higher potency of CR8 when compared to roscovitine, we selected an 
Fig. 6 Central administration of CR8 reduces lesion size following traumatic brain injury (TBI). (a) Unbiased stereological assessment of lesion volume at 21 days post-TBI was performed on cresyl violet stained brain sections. (b) Lesion quantification. CR8 treatment significantly reduced the lesion size at 21 days post-TBI $\left({ }^{+++} p<0.001\right.$ $v s$ vehicle). Analysis by onetailed paired Student's $t$ test versus sham and vehicle-treated groups. Mean \pm standard error of the mean ( $n=10$ /group). (c) Lining TBI-induced lesion volume test at 21 days post-TBI. Linear regression model ( $\mathrm{n}=5 /$ group) followed by determination of statistical significance and coefficient of correlation $(p<0.0001$; $r^{2}=0.96$ ) ear regression analysis comparwith foot faults in the beam walk
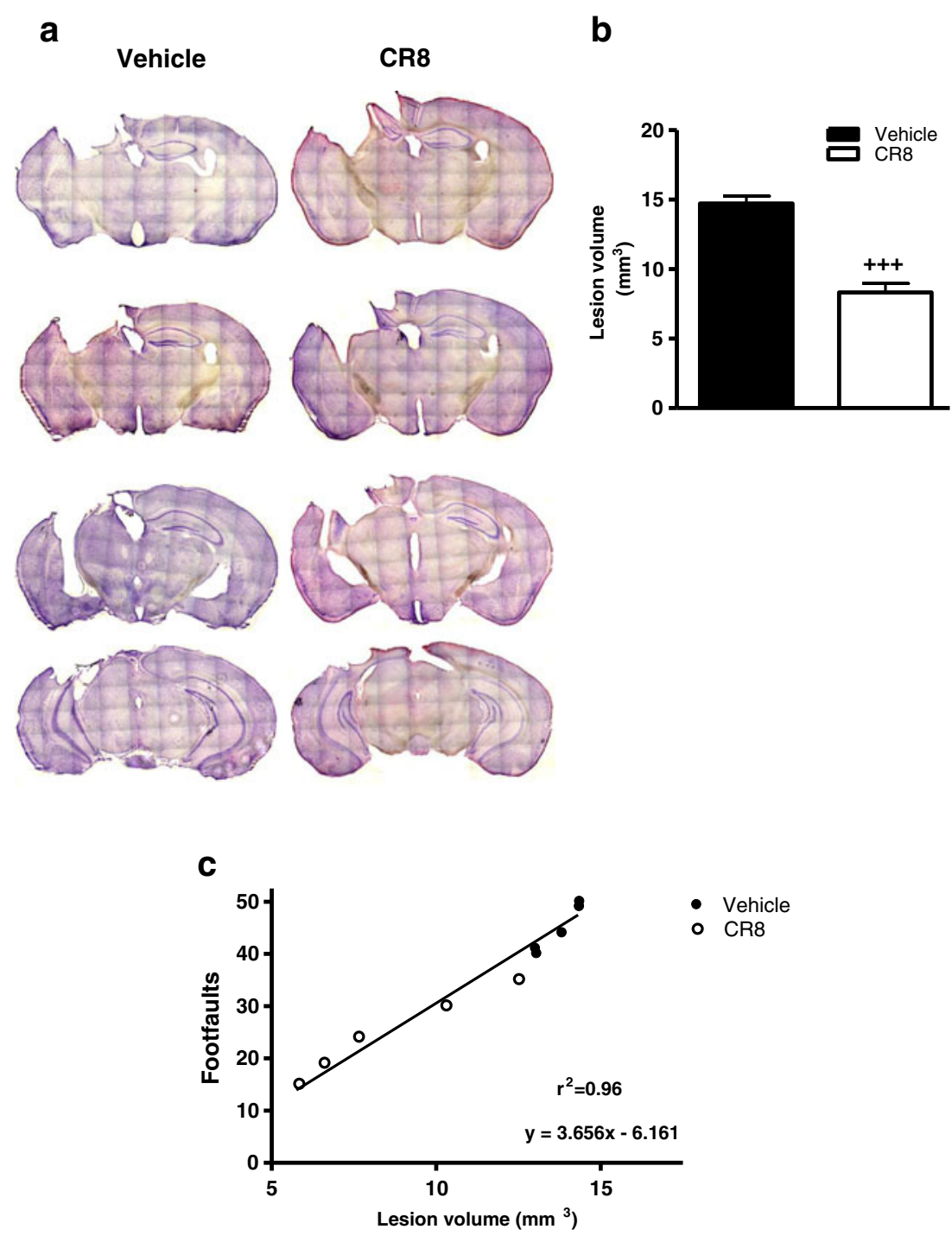

intracerebroventricular dose of CR8 that was 20 times, and an intraperitoneal dose that was 10 times, less than previously tested effective dose of roscovitine [18].

CCI increased expression of cyclins $\mathrm{A}$ and $\mathrm{B} 1$ at $6 \mathrm{~h}$ postinjury followed by the elevation of phospho-(Ser) CDKs substrate, indicating general activation of CDKs. In addition, elevated phospho-(Ser54) n-myc levels, indicating specific CDK1 activation, were detected at $6 \mathrm{~h}$ post-injury. Cyclin A has been shown to regulate CDK1 through direct binding or CDK2-dependent mechanisms, leading to phosphorylation of $\mathrm{Rb}[38,39]$. Increased levels of cyclin A have been observed in models of CNS injury and neuronal apoptosis [18, 40]. The continuation of G2 phase and G2/M transition is induced by accumulation of B-type cyclins and their association with CDK1 [7, 10]. Elevated cyclin B1 expression has been observed in models of Alzheimer's disease, spinal cord injury, and neuronal apoptosis [4144]. Our data demonstrate that TBI robustly induces CCA markers and that $\mathrm{CR} 8$ treatment significantly reduces CCA pathways. Furthermore, our data show elevated levels of $145 / 150 \mathrm{kDa}$ fodrin cleavage product at $6 \mathrm{~h}$ post-injury. Fodrin, also known as spectrin, is a high molecular weight $(240 \mathrm{kDa})$ cytoskeletal protein that undergoes degradation catalyzed by activated proteases during apoptosis [26, 27]. The cleavage of the $145 / 150 \mathrm{kDa}$ fragment is both calpain(145 kDa) and caspase- (150 kDa) mediated [45]. CR8 treatment significantly decreased the levels of the 145/ $150 \mathrm{kDa}$ fragment in a manner that was temporally correlated with the inhibition of CCA, suggesting a connection between cell cycle and apoptosis in the brain after TBI.

To simulate a more clinically relevant treatment paradigm, we administered CR 8 at $3 \mathrm{~h}$ post-injury, and we investigated the long-term neuroprotective effects of the drug on TBI-induced neurological deficits and neurodegeneration. In the first study, CR8 was administered intracerebroventricularly to ensure tissue access. CR8-treated mice 
Fig. 7 Central administration of CR8 improves neuronal survival in the CA3 and DG after traumatic brain injury (TBI). (a, b) Unbiased stereological quantification of neuronal cell loss in the CA3 and dentate gyrus (DG) subregions of the hippocampus at 21 days post-TBI. Significant neuronal cell loss in the CA3 and DG caused by TBI $\left({ }^{* *} p<0.01 v s\right.$ sham). CR8 treatment significantly improved survival of neurons in the CA3 (a) and DG (b) regions $\left({ }^{++} p<0.01 v s\right.$ vehicle). Analysis by one-way analysis of variance, followed by post-hoc adjustments using the Student-Newman-Keuls test. Mean \pm standard error of the mean ( $\mathrm{n}=5 /$ group). (c) Representative images of cresyl violetstained CA3 hippocampal subregion from brain sections of vehicle- and CR8-treated TBI groups illustrate improved neuronal survival by CR8 treatment. (d) Linear regression analysis comparing TBI-induced neuronal loss in the CA3 with latency to reach the submerged platform on post-injury day 17 of the Morris water maze test followed by determination of statistical significance and coefficient of correlation $\left(p<0.0001 ; \mathrm{r}^{2}=0.94\right)$. (e) Linear regression analysis comparing TBI-induced neuronal loss in the CA3 with "discrimination index" (\%) in NOR test followed by determination of statistical significance and coefficient of correlation $\left(p<0.0001 ; r^{2}=0.96\right)$. (f) Linear regression analysis comparing TBI-induced neuronal loss in the DG with latency to reach the submerged platform on postinjury day 17 of the MWM followed by determination of statistical significance and coefficient of correlation ( $\mathrm{p}<$ $0.0001 ; r^{2}=0.95$ ). (g) Linear regression analysis comparing TBI-induced neuronal loss in the DG with DI (\%) in the novel object recognition test followed by determination of statistical significance and coefficient of correlation $\left(p<0.0001 ; r^{2}=0.96\right)$ a

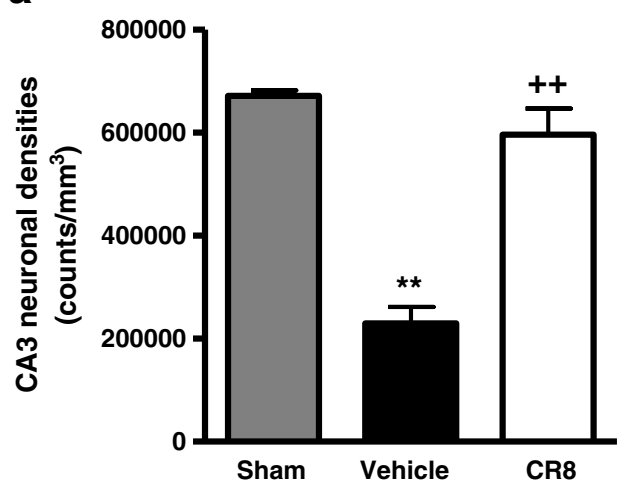

b

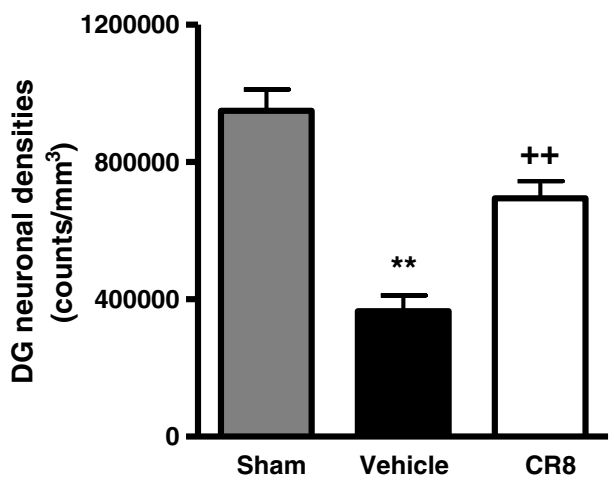

C

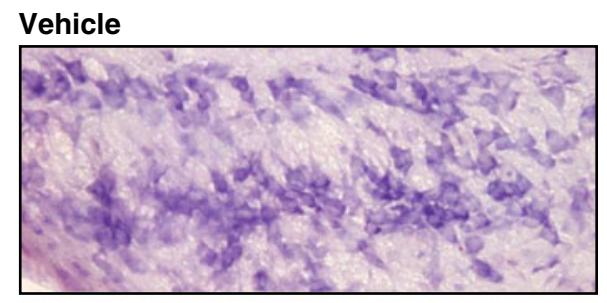

CR8
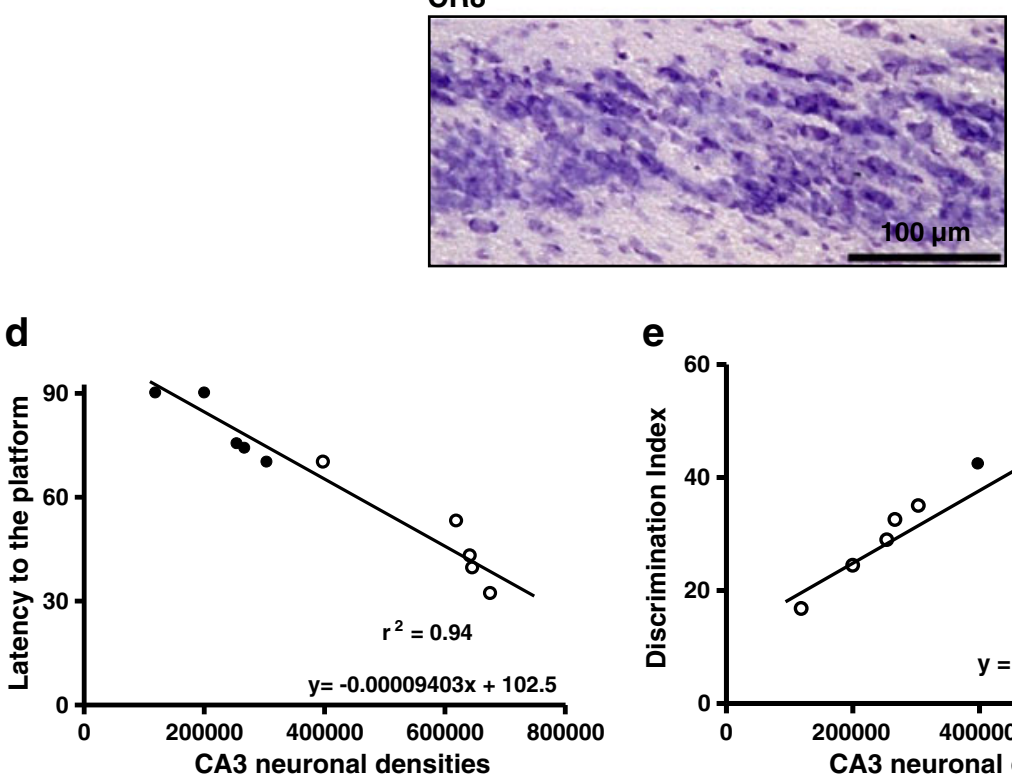

e

f

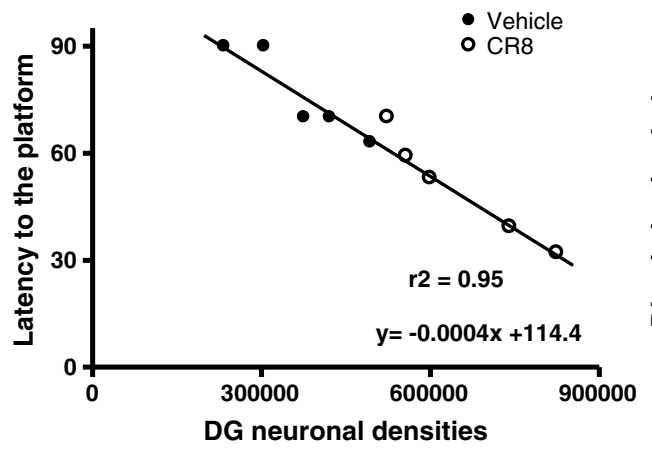

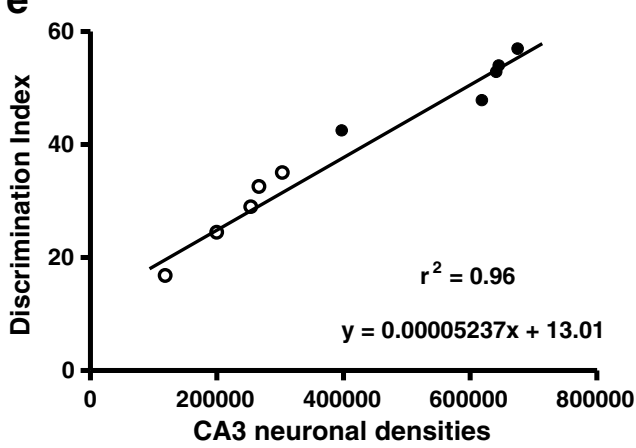

g

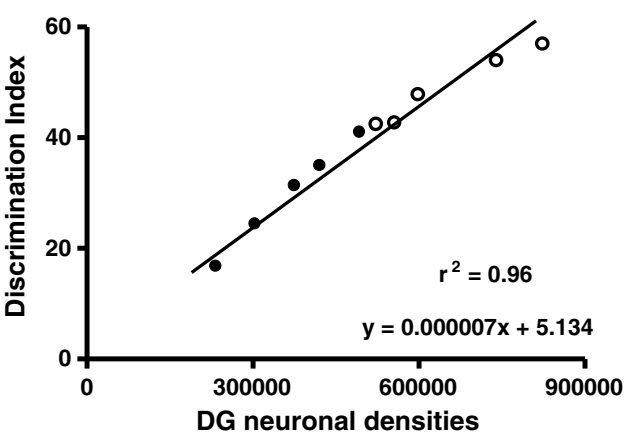


Fig. 8 Central administration of CR8 attenuates neuronal degeneration and neuronal cell loss in the cortex and thalamus after traumatic brain injury (TBI). (a, b) Qualitative assessment of neuronal degeneration at $24 \mathrm{~h}$ post-TBI using Fluoro-Jade B staining. Representative confocal images from vehicle-treated mice (a) demonstrate degenerating neurons (Fluoro-Jade B positive) around the lesion injury site (2), and in subcortical regions (1), whereas CR8-treated mice (b) had fewer Fluoro-Jade $\mathrm{B}$ positive neurons at the injury site (4), and in subcortical areas (3), indicating attenuated neuronal degeneration. Higher magnification images from the indicated regions are shown ( $\mathrm{n}=3$ /group). (c, d) Representative images of cresyl violetstained brain sections illustrate morphological features of neurons, and improved neuronal survival in the cortex (c) and thalamus (d) by CR8 treatment at 21 days after injury. (e) Unbiased stereological quantification of neuronal cell loss in the cortex at 21 days post-TBI. CR8 treatment attenuated TBI-induced $\left({ }^{* *} p<0.01 v s\right.$ sham) neuronal loss in the cortex $\left({ }^{+} p<0.05 v s\right.$ vehicle) when compared to the vehicle-treated TBI group. (f) Unbiased stereological quantification of neuronal cell loss in the thalamus at 21 days post-TBI.

CR8-treated TBI mice exhibited significantly improved neuronal survival in the thalamus $\left({ }^{+++} p<\right.$ $0.001 v s$ vehicle) when compared with vehicle-treated TBI mice $\left({ }^{* *} p<0.01 v s\right.$ sham). Analysis by one-way analysis of variance followed by post-hoc adjustments using the Student-

Newman-Keuls test. Mean \pm standard error of the mean $(\mathrm{n}=5 /$ group $)$
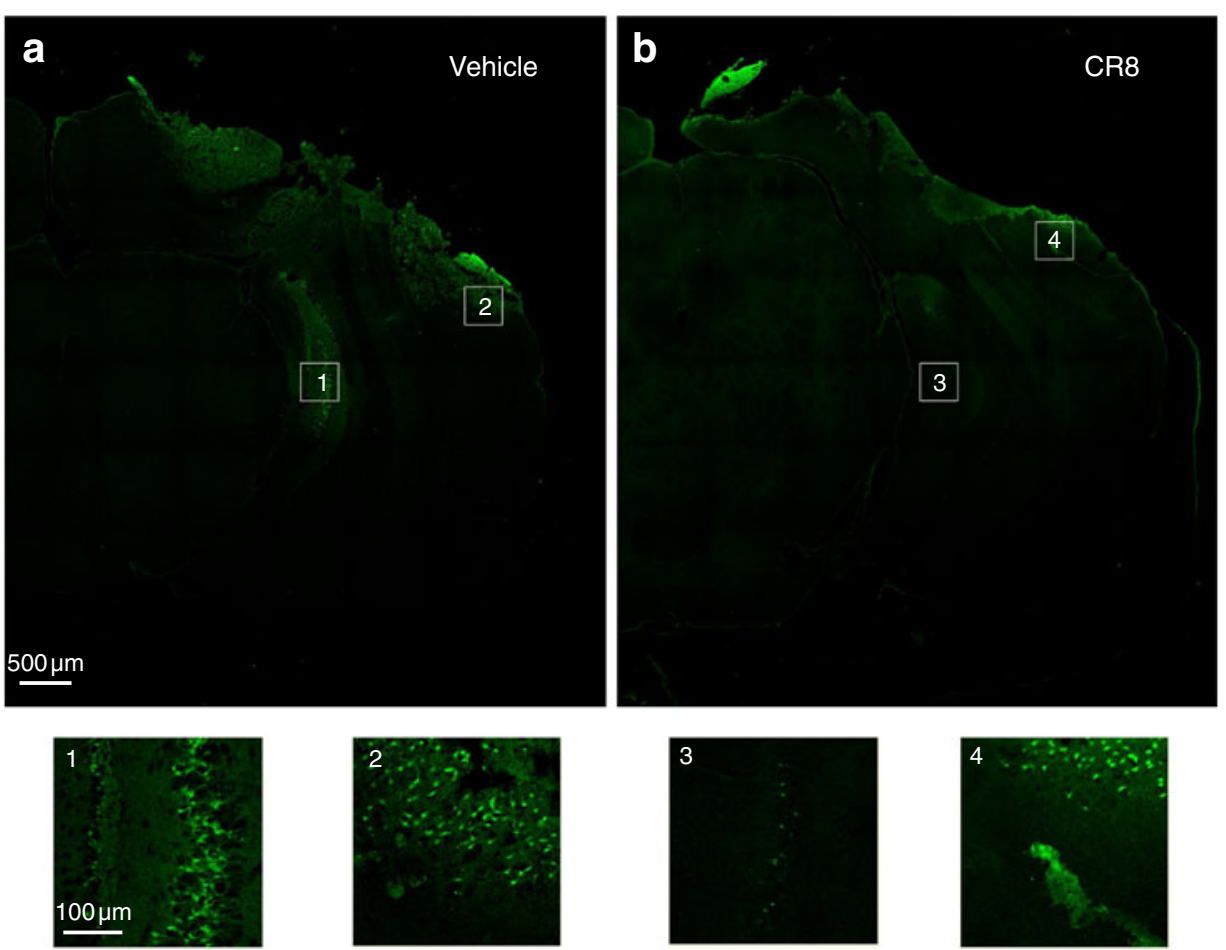

C
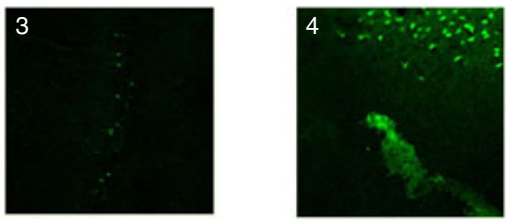

d

\section{Cortex}
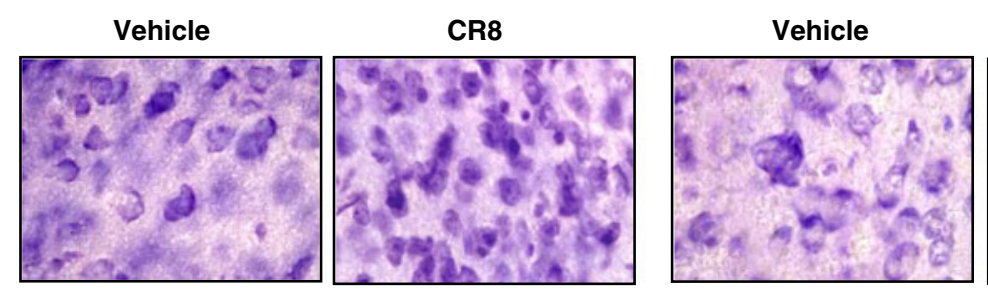

CR8

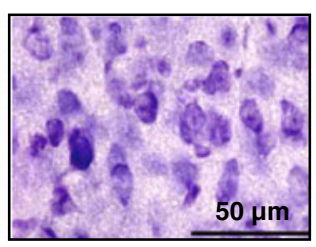

e

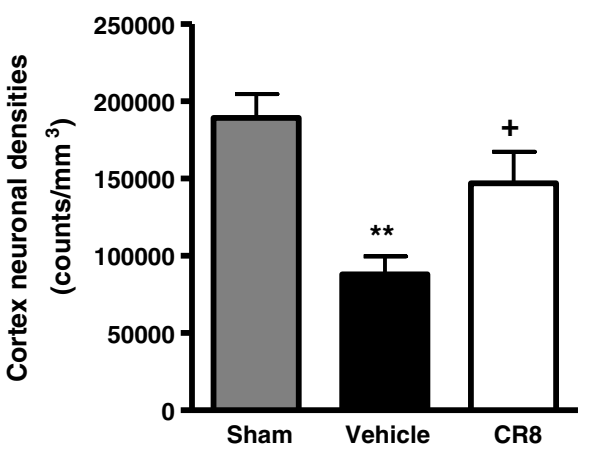

f

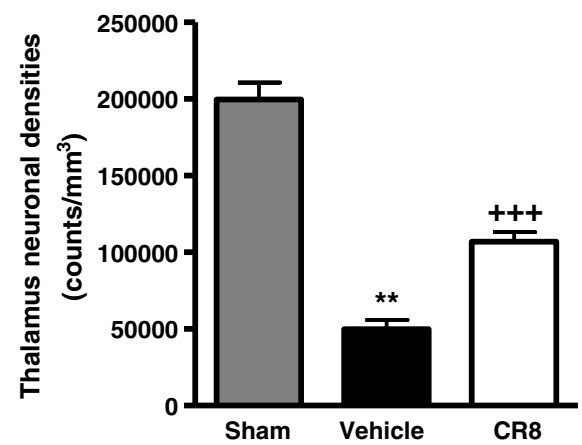

showed markedly improved sensorimotor outcomes (a measure of cortical function) compared to vehicle-treated groups. TBI-induced gait and hind-limb stride deficits result in foot faults in the beam walk test, and reflect lesions in sensorimotor cortex [46]. The volume of the cortical lesion serves as a reliable marker for tissue loss and neurodegeneration. Unbiased stereology showed a significant reduction in lesion volume in CR8-treated mice compared to the vehicletreated controls. The extent of locomotor impairment after injury to the sensorimotor cortex is well correlated with lesion size [18]. In these studies, regression analysis demonstrated a strong and highly significant correlation between improved motor function and reduced lesion size following CR8 administration. 
To assess neuronal degeneration and its modulation by CR8 treatment, we performed Fluoro-Jade B staining at $24 \mathrm{~h}$ after injury. TBI caused extensive neurodegeneration, as indicated by dense Fluoro-Jade B positive neurons, not only around the lesion site, but also within the subcortical region. These data are in agreement with previous studies that suggest that ischemia- and CCI-induced damage to the cortex may cause retrograde degeneration of neurons in subcortical regions [47, 48]. CR8 treatment attenuated neuronal degeneration, as indicated by fewer Fluoro-Jade B positive neurons compared to vehicle-treated controls. We previously quantified TBI-induced progressive neuronal loss in the cortex after CCI using unbiased stereological techniques and we found that survival of cortical neurons was enhanced following cell cycle inhibition $[18,23]$. Here we expanded our inquiry to include the thalamus. We demonstrate considerable neuronal loss in the cortex and thalamus at 21 days after CCI. Importantly, the trauma-induced neuronal cell loss in the cortex, as well as in the thalamus was significantly attenuated by a delayed administration of a single dose of CR8.

Cognitive performance in the MWM and NOR, in part, reflect hippocampal integrity $[49,50]$. The parameters assessed in the acquisition phase and probe trial evaluate spatial learning and reference memory [51]. In addition, improved performance across the acquisition trials of the MWM can be assessed by swimming strategies or paths used by the mice to locate the platform [24]. We have previously used similar search strategy analysis for demonstrating spatial/nonspatial learning impairments in the Barnes circular maze following CCI in mice [52]. In the MWM test, these strategies are classified as: spatial, if the animal swims directly to the platform; systematic, if the animal searches the interior portion or the entire tank without any bias toward a particular part of the tank; and looping, if the animal circles around the periphery demonstrating "thigmotaxis" or "wall-hugging" behavior [24]. Improved cognitive performance, in terms of overall search strategy being used, is indicated by a switch from looping to spatial swimming patterns across trials. The object exploration and discrimination trials of the NOR task assess retention or intact memory [25]. CCI caused important and widespread cognitive impairment in spatial learning, reference and retention memory. CR8 treatment significantly improved each of these cognitive aspects. Furthermore, CR8-treated mice preferably used spatial or systematic swimming strategies in comparison to a looping pattern to locate the platform on the final day of the acquisition phase.

We have previously demonstrated that CCI causes hippocampal neuronal cell loss and that roscovitine treatment significantly, albeit modestly, improves neuronal survival in the DG [18]. Here, neuronal loss in both CA3 and DG was significantly attenuated by $\mathrm{CR} 8$ treatment. The relative
Fig. 9 Systemic administration of CR8 improves cognitive function after traumatic brain injury (TBI). (a) Fine motor coordination deficits were quantified using the beam walk test. TBI induced significant cognitive impairments on post-injury day $17\left({ }^{* * *} p<0.001\right.$ vs sham). CR8 treatment failed to cause significant improvement in motor performance. (b) Spatial learning and memory was assessed using the Morris water maze (MWM) test. The factors of "post-injury days" $(\mathrm{F}(3,84)=10.125)(p<0.001)$ and "groups" $(\mathrm{F}(2,84)=7.931)(p<$ $0.001)$ were statistically significant. TBI induced significant cognitive impairments on post-injury day $17\left({ }^{* *} p<0.01 \mathrm{vs}\right.$ sham $)$. CR8-treated TBI mice had reduced latency to locate the submerged platform at day 17 post-TBI $\left({ }^{+} p=0.022 v s\right.$ vehicle) when compared to vehicle-treated TBI mice. Analysis by repeated measures one-way analysis of variance followed by post-hoc adjustments using the Student-Newman-Keuls test. (c) Reference memory was assessed using the probe trial of the MWM test. TBI caused significant cognitive impairments in this test $\left({ }^{* *} p<0.01\right.$ and ${ }^{*} p<0.05$ vs sham). CR8-treated TBI mice exhibited significant cognitive improvements in terms of number of entries $\left({ }^{++} p<0.01 v s\right.$ vehicle). Analysis by one-way analysis of variance followed by post-hoc adjustments using the Student-Newman-Keuls test. (d) Cognitive performance was further evaluated by swim strategy analysis. Vehicle-treated TBI mice exhibited higher reliance on looping strategies rather than spatial and systematic patterns $(p<0.001$ vs sham; $\left.\chi^{2}=23.34\right)$. CR8-treated TBI mice showed significantly reduced usage of looping strategy and increased reliance on spatial and systematic swim strategies $\left(p<0.001 v s\right.$ vehicle; $\left.\chi^{2}=23.34\right)$. (e) Retention or intact memory was assessed using the novel object recognition test. Vehicletreated TBI mice showed significant cognitive impairments in this test $\left({ }^{* *} p<0.01\right.$ vs sham). CR8-treated TBI mice had significant improvements in cognitive performance in terms of discrimination index $\left({ }^{++} p<\right.$ $0.01 v s$ vehicle) when compared to vehicle-treated TBI mice. Analysis by one-way analysis of variance followed by post-hoc adjustments using the Student-Newman-Keuls test. Mean \pm standard error of the mean $(n=7-8 /$ group $)$

contribution and significance of different subregions of the hippocampus in encoding and retrieval of learning and memory function can be determined by counting neuronal cells in these regions [53-55]. Lesions to CA3 and DG subregions have been found to be associated with cognitive impairment in spatial navigation and object exploration tasks [54-58]. Here, we demonstrate statistically significant positive correlations between improved cognitive performance in both the MWM and NOR tests after CR8 treatment with increased neuronal survival in the $\mathrm{CA} 3$ and $\mathrm{DG}$ regions.

Using a smaller group of animals, we also performed a proof of principle study to examine the effect of delayed systemic administration of CR8, because it is a more clinically relevant route of administration. Systemic administration of CR8 significantly improved spatial learning and reference memory functions in the acquisition and probe trials of MWM test, respectively, and retention memory in the NOR test. In addition, CR8-treated mice showed a preference toward spatial or systematic swimming strategies in comparison to a looping pattern to locate the platform on the final day of acquisition phase of MWM test. Although systemic administration of CR8 was associated with fewer foot faults at all post-injury time points examined, differences 

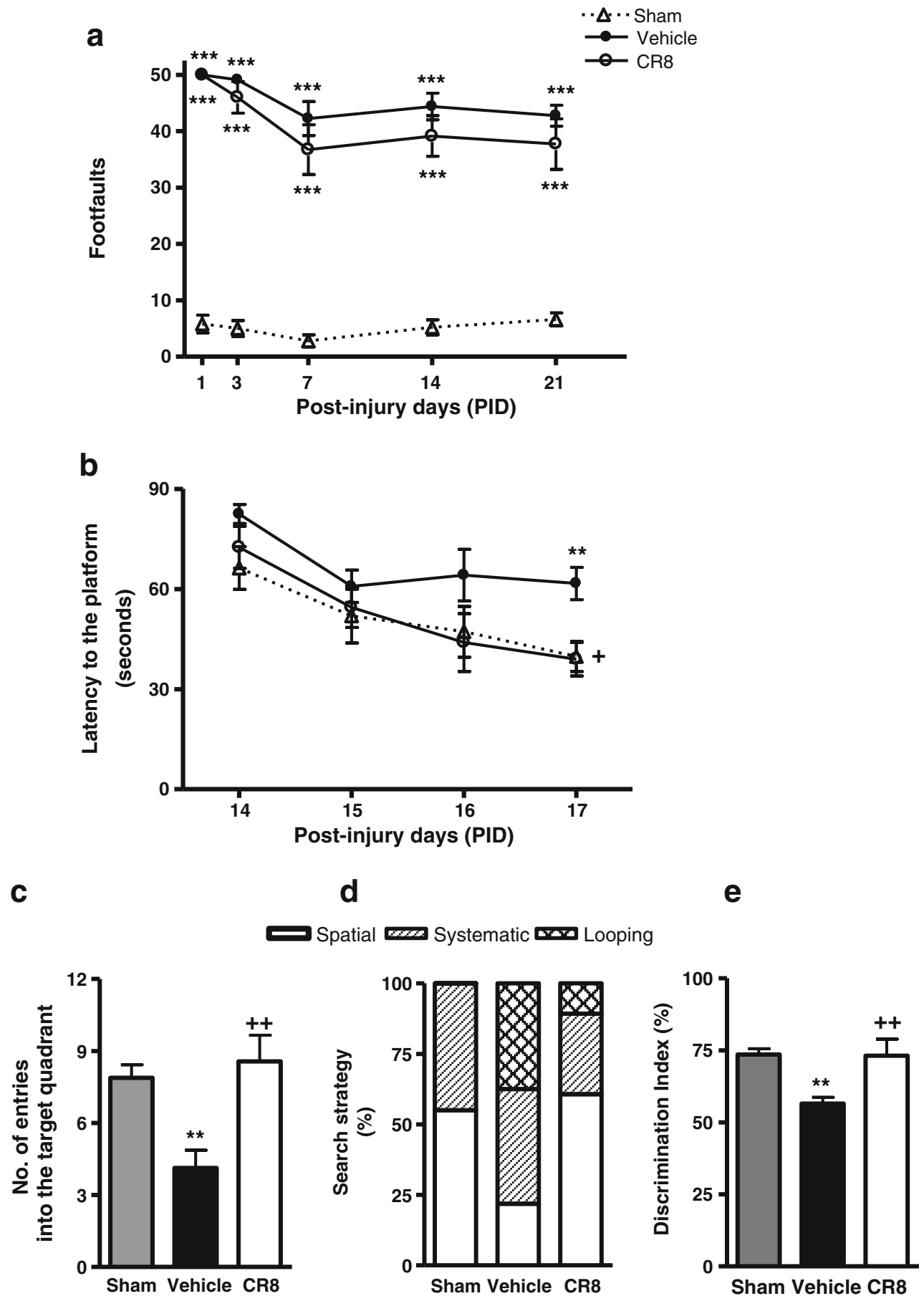

from vehicle-treated animals did not reach significance. Differences between the intraperitoneal and intracerebroventricular administration routes for this test in part likely reflected the smaller number of animals used for the intraperitoneal study and the fact that dose response studies were not performed to optimize the systemic dose required.

In conclusion, these studies demonstrate that the selective and potent $\mathrm{CDK}$ inhibitor (CR8) inhibited TBI-induced CCA and apoptotic pathways and that delayed administration of CR8 reduced behavioral impairments and neurodegeneration. The improvement in motor function by CR 8 was highly correlated with decreased lesion volume, whereas the improvement in cognitive function was strongly correlated with enhanced neuronal survival in the CA3 and DG hippocampal subregions. Our study provides further evidence that CDK inhibitors may be promising therapeutic agents for the treatment of clinical TBI. 
Acknowledgments We thank David Knipp, Stephanie Custer, and Titilola Akintola for expert technical assistance. This work was supported by a grant from the National Institutes of Health (grant No. R01 NS052568).

Required Author Forms Disclosure forms provided by the authors are available with the online version of this article.

Disclosure/Conflict of Interest The authors declare that they have no competing financial interests.

\section{References}

1. Faul M, Xu L, Wald M, Coronado V. Traumatic brain injury in the United States: Emergency department visits, hospitalizations and deaths 2002-2006. Atlanta (GA): Centers for Disease Control and Prevention, National Center for Injury Prevention and Control 2010;xx:xx-xx.

2. Dutton R, Stansbury L, Leone S, Kramer E, Hess J, Scalea T. Trauma mortality in mature trauma systems: are we doing better? An analysis trauma mortality patterns, 1997-2008. J Trauma 2010;69:620-626.

3. Panter SS, Faden AI. Pretreatment with NMDA antagonists limits release of excitatory amino acids following traumatic brain injury. Neurosci Lett. 1992;136:165-168.

4. Bramlett H, Dietrich W. Progressive damage after brain and spinal cord injury: pathomechanisms and treatment strategies. Prog Brain Res 2007;161:125-141.

5. Loane DJ, Faden AI. Neuroprotection for traumatic brain injury: translational challenges and emerging therapeutic strategies. Trends Pharmacol Sci 2010;31:596-604.

6. Kranenburg O, vanderEb A, Zantema A. Cyclin D1 is an essential mediator of apoptotic neuronal cell death. EMBO J 1996;15:4654

7. Herrup K, Yang Y. Cell cycle regulation in the postmitotic neuron: oxymoron or new biology? Nat Rev Neurosci 2007;8:368-378.

8. Osuga $\mathrm{H}$, Osuga $\mathrm{S}$, Wang $\mathrm{F}$, et al. Cyclin-dependent kinases as a therapeutic target for stroke. Proc Natl Acad Sci U S A 2000;97:10254-10259.

9. Arendt T. Synaptic plasticity and cell cycle activation in neurons are alternative effector pathways: the "Dr. Jekyll and Mr. Hyde concept" of Alzheimer's disease or disease or the yin and yang of neuroplasticity. Prog Neurobiol 2003;71:83-248.

10. Stoica BA, Byrnes KR, Faden AI. Cell cycle activation and CNS injury. Neurotox Res 2009;16:221-237.

11. Byrnes K, Faden A. Role of cell cycle proteins in CNS injury. Neurochem Res 2007;32:1799-1807.

12. Greene L, Biswas S, Liu D. Cell cycle molecules and vertebrate neuron death: E2F at the hub. Cell Death Differ 2004;11:49-60.

13. Nguyen M, Boudreau M, Kriz J, Couillard-Despres S, Kaplan D, Julien J. Cell cycle regulators in the neuron death pathway of amyotrophic lateral sclerosis caused by mutant superoxide dismutase. J Neurosci 2003;23:2131-2140.

14. Wesierska-Gadek J, Gritsch D, Zulehner N, Komina O, Maurer M. Roscovitine, a selective CDK inhibitor, reduces the basal and estrogen-induced phosphorylation of ER-alpha in human ERpositive breast cancer cells. J Cell Biochem. [Research Support, Non-U.S. Gov't]. 2011;112:761-772.

15. Komina O, Nosske E, Maurer M, Wesierska-Gadek J. Roscovitine, a small molecule CDK inhibitor induces apoptosis in multidrugresistant human multiple myeloma cells. J Exp Ther Oncol. [Research Support, Non-U.S. Gov't]. 2011;9:27-35.
16. Bettayeb K, Oumata N, Echalier A, et al. CR8, a potent and selective, roscovitine-derived inhibitor of cyclin-dependent kinases. Oncogene. [Research Support, Non-U.S. Gov't] 2008;27:5797-5807.

17. Hilton GD, Stoica BA, Byrnes KR, Faden AI. Roscovitine reduces neuronal cell loss, glial activation, and neurological deficits after brain trauma. J Cereb Blood Flow Metab 2008;28:1845-1859.

18. Kabadi SV, Stoica BA, Byrnes KR, Hanscom M, Loane DJ, Faden AI. Selective CDK inhibitor limits neuroinflammation and progressive neurodegeneration after brain trauma. J Cereb Blood Flow Metab 2011, Aug 10; doi:10.1038/jcbfm.2011.117.

19. Bettayeb K, Baunbaek D, Delehouze C, et al. CDK Inhibitors roscovitine and CR8 trigger Mcl-1 down-regulation and apoptotic cell death in neuroblastoma cells. Genes Cancer 2010;1:369-380.

20. Nutley BP, Raynaud FI, Wilson SC, et al. Metabolism and pharmacokinetics of the cyclin-dependent kinase inhibitor Rroscovitine in the mouse. Mol Cancer Ther. [Research Support, Non-U.S. Gov't] 2005;4:125-139.

21. Fox GB, Fan L, Levasseur RA, Faden AI. Sustained sensory/motor and cognitive deficits with neuronal apoptosis following controlled cortical impact brain injury in the mouse. J Neurotrauma 1998;15:599-614.

22. Loane DJ, Pocivavsek A, Moussa CE, et al. Amyloid precursor protein secretases as therapeutic targets for traumatic brain injury. Nat Med 2009;15:377-379.

23. Kabadi SV, Stoica BA, Loane DJ, et al. Cyclin D1 gene ablation confers neuroprotection in traumatic brain injury. J Neurotrauma 2011, Sep 6.

24. Brody DL, Holtzman DM. Morris water maze search strategy analysis in PDAPP mice before and after experimental traumatic brain injury. Exp Neurol 2006;197:330-340.

25. Bevins R, Besheer J. Object recognition in rats and mice: a onetrial non-matching-to-sample learning task to study "recognition memory." Nat Protoc 2006;1:1306-1311.

26. Siman R, Baudry M, Lynch G. Brain fodrin: substrate for calpain I, an endogenous calcium-activated protease. Proc Natl Acad Sci U S A 1984;81:3572-3576.

27. Siman R, McIntosh TK, Soltesz KM, Chen Z, Neumar RW, Roberts VL. Proteins released from degenerating neurons are surrogate markers for acute brain damage. Neurobiol Dis 2004;16:311-320.

28. Nauta WJH, Feirtag M, eds. Fundamental Neuroanatomy. New York: W.H. Freeman and Company; 1986.

29. Martin JH, ed. Neuroanatomy, text and atlas, 2nd ed. Stamford, CT: Appleton \& Lange; 1996.

30. Cernak I, Stoica B, Byrnes K, Di Giovanni S, Faden A. Role of the cell cycle in the pathophysiology of central nervous system trauma. Cell cycle. 2005;4:1286-1293.

31. Giovanni SD, Movsesyan V, Ahmed F, et al. Cell cycle inhibition provides neuroprotection and reduces glial proliferation and scar formation after traumatic brain injury. Proc Natl Acad Sci USA 2005; 102:8333-8338.

32. Byrnes K, Stoica B, Fricke S, Di Giovanni S, Faden A. Cell cycle activation contributes to post-mitotic cell death and secondary damage after spinal cord injury. Brain 2007;130:2977-2992.

33. Meijer L, Borgne A, Mulner O, et al. Biochemical and cellular effects of roscovitine, a potent and selective inhibitor of the cyclin-dependent kinases cdc2, cdk2 and cdk5. Eur J Biochem 1997;243:527-536.

34. Cernak I. Animal models of head trauma. NeuroRx [Review] $2005 ; 2: 410-422$.

35. Dixon CE, Clifton GL, Lighthall JW, Yaghmai AA, Hayes RL. A controlled cortical impact model of traumatic brain injury in the rat. J Neurosci Methods [Research Support, U.S. Gov't, P.H.S.] 1991;39:253-262.

36. Mori T, Kawamata T, Katayama Y, Maeda T, Aoyama N, Kikuchi T, et al. Antioxidant, OPC-14117, attenuates edema formation, and subsequent tissue damage following cortical contusion in rats. Acta Neurochir Suppl 1998;71:120-122. 
37. Faden AI, Fox GB, Di X, et al. Neuroprotective and nootropic actions of a novel cyclized dipeptide after controlled cortical impact injury in mice. J Cereb Blood Flow Metab 2003;23:355-363.

38. Mitra J, Enders GH. Cyclin A/Cdk2 complexes regulate activation of Cdk1 and Cdc25 phosphatases in human cells. Oncogene [Research Support, Non-U.S. Gov't

39. Research Support, U.S. Gov't, P.H.S.] 2004;23:3361-3367.

40. Lundberg A, Weinberg R. Functional inactivation of the retinoblastoma protein requires sequential modification by at least two distinct cyclin-CDK complexes. Mol Cell Biol 1998;23:10441053.

41. Copani A, Condorelli F, Caruso A, et al. Mitotic signaling by betaamyloid causes neuronal death. Faseb J [in vitro] 1999;13:22252234.

42. Vincent I, Jicha G, Rosado M, Dickson DW. Aberrant expression of mitotic cdc2/cyclin B1 kinase in degenerating neurons of Alzheimer's disease brain. J Neurosci [Research Support, U.S. Gov't, P.H.S.] 1997;17:3588-3598.

43. Mosch B, Morawski M, Mittag A, Lenz D, Tarnok A, Arendt T. Aneuploidy and DNA replication in the normal human brain and Alzheimer's disease. J Neurosci [Research Support, Non-U.S. Gov't] 2007;27:6859-6867.

44. Tian DS, Xie MJ, Yu ZY, et al. Cell cycle inhibition attenuates microglia induced inflammatory response and alleviates neuronal cell death after spinal cord injury in rats. Brain Res [Research Support, Non-U.S. Gov't] 2007;1135:177-185.

45. Maestre C, Delgado-Esteban M, Gomez-Sanchez JC, Bolanos JP, Almeida A. Cdk5 phosphorylates Cdh1 and modulates cyclin B1 stability in excitotoxicity. EMBO J [Research Support, Non-U.S. Gov't] 2008;27:2736-2745.

46. Saatman KE, Creed J, Raghupathi R. Calpain as a therapeutic target in traumatic brain injury. Neurotherapeutics 2010;7:31-42.

47. Goldstein LB. Model of recovery of locomotor ability after sensorimotor cortex injury in rats. ILAR J 2003;44:125-129.
48. Iizuka H, Sakatani K, Young W. Neural damage in the rat thalamus after cortical infarcts. Stroke [Research Support, Non-U.S. Gov't Research Support, U.S. Gov't, P.H.S.] 1990;21:790-794.

49. Hall ED, Sullivan PG, Gibson TR, Pavel KM, Thompson BM, Scheff SW. Spatial and temporal characteristics of neurodegeneration after controlled cortical impact in mice: more than a focal brain injury. J Neurotrauma 2005;22:252-265.

50. Redish AD, Touretzky DS. The role of the hippocampus in solving the Morris water maze. Neural Comput 1998;10:73-111.

51. Broadbent NJ, Gaskin S, Squire LR, Clark RE. Object recognition memory and the rodent hippocampus. Learn Mem 2010;17:5-11.

52. Vorhees CV, Williams MT. Morris water maze: procedures for assessing spatial and related forms of learning and memory. Nature protocols [Research Support, N.I.H., Extramural] 2006;1:848-858.

53. Fox GB, Fan L, LeVasseur RA, Faden AI. Effect of traumatic brain injury on mouse spatial and nonspatial learning in the Barnes circular maze. J Neurotrauma 1998;15:1037-1046.

54. Lee I, Hunsaker MR, Kesner RP. The role of hippocampal subregions in detecting spatial novelty. Behav Neurosci 2005;119:145-153.

55. Lee I, Kesner R. Encoding versus retrieval of spatial memory: double dissociation between the dentate gyrus and the perforant path inputs into CA3 in the dorsal hippocampus. Hippocampus 2004;14:66-76.

56. Gilbert PE, Brushfield AM. The role of the CA3 hippocampal subregion in spatial memory: a process oriented behavioral assessment. Prog Neuropsychopharmacol Biol Psychiatry 2009;33:774-781.

57. Brunson KL, Eghbal-Ahmadi M, Bender R, Chen Y, Baram TZ. Long-term, progressive hippocampal cell loss and dysfunction induced by early-life administration of corticotropin-releasing hormone reproduce the effects of early-life stress. Proc Natl Acad Sci U S A [Research Support, U.S. Gov't, P.H.S.] 2001;98:8856-8861.

58. Jerman T, Kesner RP, Hunsaker MR. Disconnection analysis of CA3 and DG in mediating encoding but not retrieval in a spatial maze learning task. Learn Mem 2006;13:458-464. 